\title{
Decays of charged $B$ mesons into three charged leptons and a neutrino
}

\author{
A. Danilina, ${ }^{1,2,3}$ N. Nikitin, ${ }^{1,2,3}$ and K. Toms ${ }^{4}$ \\ ${ }^{1}$ Lomonosov Moscow State University, Department of Physics, Moscow 119991, Russia \\ ${ }^{2}$ Lomonosov Moscow State University, Skobeltsyn Institute of Nuclear Physics, Moscow 119991, Russia \\ ${ }^{3}$ Institute for Theoretical and Experimental Physics, Moscow 117218, Russia \\ ${ }^{4}$ Department of Physics and Astronomy, University of New Mexico, Albuquerque, New Mexico 87106, USA
}

(Received 9 November 2019; accepted 30 April 2020; published 11 May 2020)

\begin{abstract}
In the framework of the Standard Model, we present predictions for partial widths, double and single differential distributions, and forward-backward lepton asymmetries for four-leptonic decays $B^{-} \rightarrow \mu^{+} \mu^{-} \bar{\nu}_{e} e^{-}, B^{-} \rightarrow e^{+} e^{-} \bar{\nu}_{\mu} \mu^{-}, B^{-} \rightarrow \mu^{+} \mu^{-} \bar{\nu}_{\mu} \mu^{-}$, and $B^{-} \rightarrow e^{+} e^{-} \bar{\nu}_{e} e^{-}$. We consider the contributions of virtual photon emission from the light and heavy quarks of the $B^{-}$meson, and we include bremsstrahlung of a virtual photon from the charged lepton in the final state. We use the model of vector meson dominance for calculation of virtual photon emission by the light quark of the $B^{-}$meson and take into account the isotopic correction. The dependence of branchings on the relative phase of the contributions of the light intermediate vector mesons is studied.
\end{abstract}

DOI: 10.1103/PhysRevD.101.096007

\section{INTRODUCTION}

Four-leptonic decays of $B$ mesons allow a precise test of Standard Model (SM) predictions in the higher orders of perturbation theory. At the same time, these decays may be background processes to the helicity-suppressed ultrarare decays $B_{d, s} \rightarrow \mu^{+} \mu^{-}$, which are under study at the Large Hadron Collider (LHC) [1-5]. These studies are motivated by searches for Beyond the Standard Model physics.

Rare four-leptonic decays of $B$ mesons in the SM may be divided into two groups. The decays of the first group are forbidden at the tree level and occur through the higher order loop diagrams of perturbation theory- "penguin" and/or "box." In this way, the SM includes flavor changing neutral currents. An example of the first group of decays is the process $B_{s} \rightarrow e^{+} e^{-} \mu^{+} \mu^{-}$and any other four-leptonic decays of neutral $B$ mesons. In the second group, in order to obtain the given multilepton final state, a number of tree level weak and electromagnetic processes are involved. Examples are the decay $B^{-} \rightarrow e^{+} e^{-} \bar{\nu}_{\mu} \mu^{-}$and analogous processes involving charged $B$ mesons. Both groups are studied at the LHC and potentially could be investigated at the Belle II experiment. Currently, only upper limits for branching ratios of the decays $B_{d, s} \rightarrow \mu^{+} \mu^{-} \mu^{+} \mu^{-}$and $B^{-} \rightarrow \mu^{+} \bar{\nu}_{\mu} \mu^{-} \mu^{-}$are available [6-8].

Published by the American Physical Society under the terms of the Creative Commons Attribution 4.0 International license. Further distribution of this work must maintain attribution to the author(s) and the published article's title, journal citation, and DOI. Funded by SCOAP ${ }^{3}$.
The experimental upper limits [6,7] for the decays $B_{d, s} \rightarrow \mu^{+} \mu^{-} \mu^{+} \mu^{-}$are an order of magnitude higher than the corresponding theoretical predictions [9] and estimates [10]. The situation with the decay $B^{-} \rightarrow \mu^{+} \bar{\nu}_{\mu} \mu^{-} \mu^{-}$is different. The experimental upper limit [8],

$$
\operatorname{Br}\left(B^{-} \rightarrow \mu^{+} \bar{\nu}_{\mu} \mu^{-} \mu^{-}\right)<0.16 \times 10^{-7},
$$

obtained with $95 \%$ confidence level (CL) is almost an order of magnitude lower than the theoretical predictions $[10,11]$. We present here to more detailed calculation of the branching ratios of $B^{-} \rightarrow \mu^{+} \mu^{-} \bar{\nu}_{e} e^{-}, B^{-} \rightarrow e^{+} e^{-} \bar{\nu}_{\mu} \mu^{-}$, $B^{-} \rightarrow \mu^{+} \mu^{-} \bar{\nu}_{\mu} \mu^{-}$, and $B^{-} \rightarrow e^{+} e^{-} \bar{\nu}_{e} e^{-}$, taking into account isotopic effects. Also, in the phase space of the decays, a correction to nonzero lepton mass is considered. While this leads to better agreement between theory and experiment, some discrepancy remains. Special attention is given to the predictions of the behavior of differential distributions, e.g., forward-backward lepton asymmetries.

This article is organized as follows. In Sec. I, we give a task description. In Sec. II, we write the effective Hamiltonian and give definite the hadronic form factors. In Sec. III, the common dependence of the decay amplitudes $B^{-} \rightarrow \ell^{+} \ell^{-} \bar{\nu}_{\ell^{\prime}} \ell^{\prime-}$ on dilepton four-momenta is studied. Section IV contains the exact formulae for amplitudes of the decay $B^{-} \rightarrow \ell^{+} \ell^{-} \bar{\nu}_{\ell^{\prime}} \ell^{\prime-}$ for $\ell \neq \ell^{\prime}$, and Sec. V provides analogous formulas for $\ell \equiv \ell^{\prime}$. In Sec. VI, we present numerical results for the decays of charged $B$ mesons into three charged leptons and a neutrino and discuss the precision of the predictions. Section VII contains the main outcome of the work. Appendix A contains some formulas necessary for analytical calculations of 
differential distributions. Some details of the four-leptonic decay kinematics are given in Appendix B.

\section{EFFECTIVE HAMILTONIAN AND HADRONIC MATRIX ELEMENTS}

In terms of fundamental quark and lepton fields, the Hamiltonian for calculation of the amplitudes of fourlepton decays $B^{-} \rightarrow \ell^{+} \ell^{-} \bar{\nu}_{\ell^{\prime}} \ell^{\prime-}$ has the form

$$
\mathcal{H}_{\text {eff }}(x)=\mathcal{H}_{W}(x)+\mathcal{H}_{\mathrm{em}}(x) .
$$

The Hamiltonian of the transitions $b \rightarrow u W^{-} \rightarrow u \ell^{-} \bar{\nu}_{\ell}$ is written as

$$
\begin{aligned}
\mathcal{H}_{W}(x)= & -\frac{G_{F}}{\sqrt{2}} V_{u b}\left(\bar{u}(x) \gamma^{\mu}\left(1-\gamma^{5}\right) b(x)\right) \\
& \times\left(\bar{\ell}(x) \gamma_{\mu}\left(1-\gamma^{5}\right) \nu_{\ell}(x)\right)+\text { H.c. }
\end{aligned}
$$

where $u(x)$ and $b(x)$ are quark fields, $\ell(x)$ and $\nu_{\ell}(x)$ are lepton fields, $G_{F}$ is the Fermi constant, $V_{u b}$ is the corresponding matrix element of the CabibboKobayashi-Maskawa (CKM) matrix, and the matrix $\gamma^{5}$ is defined as $\gamma^{5}=i \gamma^{0} \gamma^{1} \gamma^{2} \gamma^{3}$.

The Hamiltonian of the electromagnetic interaction has the form

$$
\mathcal{H}_{\mathrm{em}}(x)=-e \sum_{f} Q_{f}\left(\bar{f}(x) \gamma^{\mu} f(x)\right) A_{\mu}(x)=-j_{\mathrm{em}}^{\mu}(x) A_{\mu}(x),
$$

where the unitary charge $e=|e|$ is normalized by $e^{2}=4 \pi \alpha_{e m} ; \alpha_{e m} \approx 1 / 137$, the fine structure constant, $Q_{f}$ is the charge of the fermion of flavor $f$ in units of the unitary charge, $f(x)$ is the fermionic field of flavor $f$, and $A_{\mu}(x)$ is the four-potential of the electromagnetic field.

We define the following nonzero hadronic matrix elements, which are needed for the subsequent calculations:

$$
\begin{aligned}
\left\langle 0\left|\bar{u} \gamma^{\mu} \gamma^{5} b\right| B^{-}\left(M_{1}, p\right)\right\rangle & =i f_{B_{u}} p^{\mu}, \\
\left\langle 0\left|\bar{q} \gamma^{\mu} Q\right| V\left(M_{V}, k, \epsilon\right)\right\rangle & =\epsilon^{\mu} M_{V} f_{V} \\
\left\langle V\left(M_{2}, q, \epsilon\right)\left|\bar{u} \gamma_{\mu} b\right| B^{-}\left(M_{1}, p\right)\right\rangle & =\frac{2 V\left(k^{2}\right)}{M_{1}+M_{2}} \varepsilon_{\mu \nu \alpha \beta} \epsilon^{* \nu} p^{\alpha} q^{\beta} \\
\left\langle V\left(M_{2}, q, \epsilon\right)\left|\bar{u} \gamma_{\mu} \gamma^{5} b\right| B^{-}\left(M_{1}, p\right)\right\rangle & =i \epsilon^{* \nu}\left[\left(M_{1}+M_{2}\right) A_{1}\left(k^{2}\right) g_{\mu \nu}-\frac{A_{2}\left(k^{2}\right)}{M_{1}+M_{2}}(p+q)_{\mu} p_{\nu}-\frac{2 M_{2}}{k^{2}}\left(A_{3}\left(k^{2}\right)-A_{0}\left(k^{2}\right)\right) k_{\mu} p_{\nu}\right], \\
\left\langle B^{*-}\left(M_{B^{*}}, k, \epsilon\right)\left|\bar{b} \gamma^{\mu} b\right| B^{-}\left(M_{1}, p\right)\right\rangle & =\frac{2 V_{b}\left(q^{2}\right)}{M_{1}+M_{B^{*}}} \varepsilon_{\mu \nu \alpha \beta} \epsilon^{* \nu} p^{\alpha} k^{\beta}
\end{aligned}
$$

where $M_{1}-B^{-}$is the meson mass, $p^{\mu}$ is the its fourmomentum, $M_{B *}$ is the $B^{*-}$ meson mass, $M_{2}$ is mass of the light $\left(\rho^{0}(770)\right.$ or $\left.\omega(782)\right)$ mesons, $M_{V}=\left\{M_{2}, M_{B^{*}}\right\}$ are masses of the intermediate vector mesons, and $\epsilon^{\mu}$ are their polarizations. Four vectors $p^{\mu}, q^{\mu}$, and $k^{\mu}$ satisfy the conservation law $p^{\mu}=q^{\mu}+k^{\mu}$. The components of the fully antisymmetric tensor $\varepsilon^{\mu \nu \alpha \beta}$ are fixed by the condition $\varepsilon^{0123}=-\varepsilon_{0123}=-1$, and $g_{\mu \nu}$ is the metric tensor in Minkowsky space with $\operatorname{diag} g_{\mu \nu}=(1,-1,-1,-1)$.

\section{GENERIC STRUCTURE OF THE AMPLITUDES FOR THE DECAYS $B^{-} \rightarrow \ell^{+} \ell^{-} \bar{\nu}_{\ell^{\prime}} \ell^{\prime-}$ WITH THE ZERO LEPTON MASS APPROXIMATION}

There are three main types of diagrams needed for description of the decays $B^{-}(p) \rightarrow \gamma^{*}(q) W^{-}(k) \rightarrow$ $\ell^{+}\left(k_{1}\right) \ell^{-}\left(k_{2}\right) \bar{\nu}_{\ell^{\prime}}\left(k_{3}\right) \ell^{\prime-}\left(k_{4}\right)$, when the flavor of lepton $\ell$ is different from the flavor of lepton $\ell^{\prime}$. The first type arises in the situation when a virtual photon is emitted by light a $u$ quark (see Fig. 1). The second type corresponds to the emission of a virtual photon from a $b$ quark (see Fig. 2). The third type is related to bremsstrahlung, when a virtual photon is emitted by the lepton $\ell^{\prime-}$ in the final state

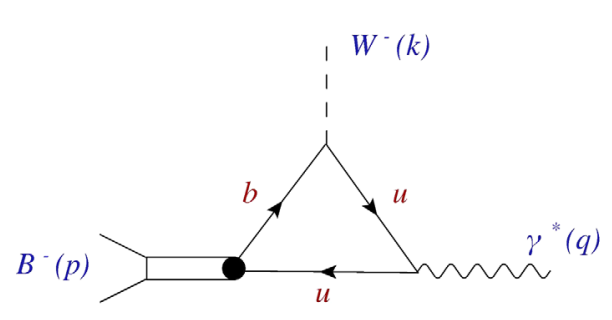

FIG. 1. Emission of a virtual photon by the light quark of the $B^{-}$meson.

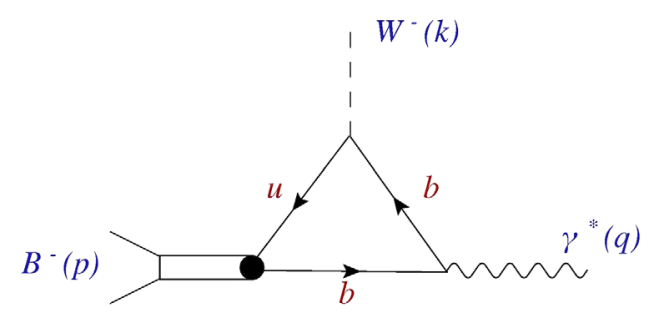

FIG. 2. Emission of a virtual photon by the heavy quark of the $B^{-}$meson. 


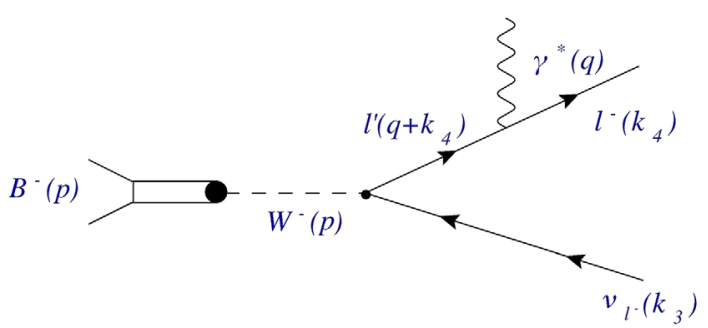

FIG. 3. Bremsstrahlung of the virtual photon.

(see Fig. 3 below). The four-momenta $q$ and $k$ are defined in Appendix B.

The structure of the amplitude, corresponding to diagrams on Figs. 1-3, may be presented as

$$
\mathcal{M}_{f i}\left(q^{2}, k^{2}\right) \sim \frac{1}{q^{2}} T_{\nu \mu}(q, k) j^{\nu}\left(k_{2}, k_{1}\right) J^{\mu}\left(k_{4}, k_{3}\right),
$$

where

$$
\begin{aligned}
T^{\nu \mu}(q, k)= & i \int d^{4} x e^{i(q x)}\langle 0| j_{e m}^{\nu}(x), \bar{u}(0) \\
& \times \gamma^{\mu}\left(1-\gamma_{5}\right) b(0)\left|B^{-}\left(M_{1}, p\right)\right\rangle \\
= & T_{\nu \mu}^{(u)}(q, k)+T_{\nu \mu}^{(b)}(q, k)+T_{\nu \mu}^{(\text {brem })}(q, k) .
\end{aligned}
$$

The lepton currents are

$$
\begin{aligned}
j^{\nu}\left(k_{2}, k_{1}\right) & =\left(\bar{\ell}\left(k_{2}\right) \gamma^{\nu} \ell\left(-k_{1}\right)\right) \quad \text { and } \\
J^{\mu}\left(k_{4}, k_{3}\right) & =\left(\overline{\ell^{\prime}}\left(k_{4}\right) \gamma^{\mu}\left(1-\gamma^{5}\right) \nu_{\ell^{\prime}}\left(-k_{3}\right)\right) .
\end{aligned}
$$

In the amplitude $\mathcal{M}_{f i}\left(q^{2}, k^{2}\right)$, the pole $1 / q^{2}$ of the photon propagator is evident. For calculations with $q^{2} \rightarrow 0$, it is necessary to take into account nonzero lepton masses. This is done using the exact formula (B4) for four-particle phase space and by introducing an effective cut for some value $q_{\min }^{2}$. If $\ell \equiv \mu$ for $q_{\min }^{2}$, it makes sense to choose the natural kinematical cut $4 m_{\mu}^{2}$. For the case when $\ell \equiv e$, it is better to use the kinematical limits of an experimental device, which are definitely higher than $4 m_{e}^{2}$.

Tensor $T_{\nu \mu}(q, k)$ satisfies the condition $q^{\nu} T_{\nu \mu}(q, k)=0$. According to this condition, and taking into account the result of Ref. [12], tensor $T_{\nu \mu}(q, k)$ has the form

$$
\begin{aligned}
T_{\nu \mu}(q, k)= & \varepsilon_{\nu \mu q k} \frac{e a\left(q^{2}, k^{2}\right)}{M_{1}}-i\left(g_{\nu \mu}-\frac{q_{\nu} q_{\mu}}{q^{2}}\right) e M_{1} b\left(q^{2}, k^{2}\right) \\
& -i e\left(k_{\nu}-\frac{(q k)}{q^{2}} q_{\nu}\right)\left(k_{\mu} \frac{2 d\left(q^{2}, k^{2}\right)}{M_{1}}-q_{\mu} \frac{2 c\left(q^{2}, k^{2}\right)}{M_{1}}\right) \\
& -i Q_{B_{u}} e f_{B_{u}} \frac{q_{\nu} k_{\mu}}{q^{2}},
\end{aligned}
$$

where $Q_{B_{u}}=Q_{b}-Q_{u}=-1$ is the electric charge of the $B^{-}$meson in units of $|e|$. The functions $a\left(q^{2}, k^{2}\right), \ldots, d\left(q^{2}, k^{2}\right)$ are dimensionless form factors which depend on two variables, the squares of the transferred four-momenta, $q^{2}$ and $k^{2}$. From (5), it follows that $d(0,0)=Q_{B_{u}} f_{B_{u}} / M_{1}$.

Using the equations of motion, in the limit of massless leptons, one can obtain the following generic structure for the amplitude $\mathcal{M}_{f i}$ :

$$
\begin{aligned}
\mathcal{M}_{f i}\left(q^{2}, k^{2}\right) \sim & \frac{e}{q^{2}}\left(\varepsilon_{\nu \mu q k} \frac{a\left(q^{2}, k^{2}\right)}{M_{1}}-i g_{\nu \mu} M_{1} b\left(q^{2}, k^{2}\right)\right. \\
& \left.+i k_{\nu} q_{\mu} \frac{2 i c\left(q^{2}, k^{2}\right)}{M_{1}}\right) j^{\nu}\left(k_{2}, k_{1}\right) J^{\mu}\left(k_{4}, k_{3}\right) .
\end{aligned}
$$

The exact calculation of the form factors $a\left(q^{2}, k^{2}\right), \ldots$, and $c\left(q^{2}, k^{2}\right)$ is quite complicated. In the current work, we will take into account only the leading singular factors to the corresponding form factors.

Let us start with a study of tensor $T_{\nu \mu}^{(u)}(q, k)$, which describes the contribution of diagram from Fig. 1 to the tensor $T_{\nu \mu}(q, k)$. The main contribution to the structure of tensor $T_{\nu \mu}^{(u)}(q, k)$ is given by the lightest intermediate vector resonances that contain a $u \bar{u}$ pair. For such states, tensor $T_{\nu \mu}^{(u)}(q, k)$ has Breit-Wigner poles for variable $q^{2}$. Taking into account only the contributions from $\rho^{0}(770)$ and $\omega(782)$ mesons, we can write

$$
\begin{aligned}
& T_{\nu \mu}^{(u)}(q, k) \rightarrow \\
& \rightarrow \sum_{i=\rho^{0}, \omega}\left\langle 0\left|\bar{u} \gamma_{\nu} u\right| V\left(M_{2 i}, q, \varepsilon\right)\right\rangle \frac{e}{M_{2 i}^{2}-q^{2}-i M_{2 i} \Gamma_{2 i}} \\
& \quad \times\left\langle V\left(M_{2 i}, q, \varepsilon\right)\left|\bar{u} \gamma_{\mu}\left(1-\gamma^{5}\right) b\right| B^{-}\left(M_{1}, p\right)\right\rangle,
\end{aligned}
$$

where $M_{2 i}$ and $\Gamma_{2 i}$ are the masses and widths, respectively, of the intermediate vector resonances.

For the zero leptonic mass approximation, the range of values of the variable $k^{2}$ is $0 \leq k^{2} \leq M_{1}^{2}$. The closest pole in $k^{2}$ is related to the appearance of the intermediate vector state $B^{*-}$. As $M_{B^{*-}}>M_{1}$, this pole lies outside of the kinematically allowed range of the decay $B^{-} \rightarrow \ell^{+} \ell^{-} \bar{\nu}_{\ell^{\prime}} \ell^{\prime-}$. The existence of the pole at the mass of the $B^{*-}$ meson is taken into account when choosing the pole parametrization of the form factors of the transitions $B \rightarrow \rho$ and $B \rightarrow \omega$ [13]. For nonzero leptonic masses, $m_{\ell^{\prime}}^{2} \leq k^{2} \leq\left(M_{1}-2 m_{\ell}\right)^{2}$. Hence, all the remarks above on the poles of tensor $T_{\nu \mu}^{(u)}$ for variable $k^{2}$ are still valid.

As the contribution from $\rho^{0}$ and $\omega$ resonances is dominant, it is possible to use the following estimate for the branching ratio of $B^{-} \rightarrow \mu^{+} \mu^{-} \bar{\nu}_{e} e^{-}$:

$$
\begin{aligned}
& \operatorname{Br}^{(u)}\left(B^{-} \rightarrow \mu^{+} \mu^{-} \bar{\nu}_{e} e^{-}\right) \\
& \approx \mid \sqrt{\operatorname{Br}\left(B^{-} \rightarrow \rho^{0} e^{-} \bar{\nu}_{e}\right) \operatorname{Br}\left(\rho^{0} \rightarrow \mu^{+} \mu^{-}\right)} \\
& \quad+\left.\sqrt{\operatorname{Br}\left(B^{-} \rightarrow \omega e^{-} \bar{\nu}_{e}\right) \operatorname{Br}\left(\omega \rightarrow \mu^{+} \mu^{-}\right)}\right|^{2} \\
& \approx 0.3 \times 10^{-7},
\end{aligned}
$$


where the necessary experimental values for the branching ratios are taken from [14]. The estimate (6) does not take into account the fact that the $\rho^{0}(770)$ meson is a wide resonance, i.e., in the case of the $\rho^{0}(770)$ meson, the naive factorization approximation should lead to a lower branching ratios. Also, in estimate (6), the photon pole, which should also lead to lower results, is not taken into account. Does estimate (6) contradict the experimental upper limit in (1)? We do not think so, because we attribute to the factor of 2 accuracy. But the estimate of (6) does point to the possibility that the minimum of the possible theoretical predictions may be above the experimental limit [8].

Now consider tensor $T_{\nu \mu}^{(b)}(q, k)$, which is related to diagram from Fig. 2. In the limit of massless leptons, there are no poles for the variable $q^{2}$ in the kinematically allowed range $0 \leq q^{2} \leq M_{1}^{2}$ for the tensor $T_{\nu \mu}^{(b)}(q, k)$. The closest pole outside the allowed range corresponds to the $b \bar{b}$ quark composition. This is the $\Upsilon(1 S)$ meson, whose mass is almost two times higher than the mass of the $B^{-}$meson. The dominant contribution to emission of a virtual photon by a heavy quark is described using the process $B^{-} \rightarrow B^{*-} \gamma^{*}$. In this case,

$$
\begin{aligned}
T_{\nu \mu}^{(b)}(q, k) \rightarrow & \left\langle 0\left|\bar{u} \gamma_{\mu}\left(1-\gamma^{5}\right) b\right| B^{*-}\left(M_{B^{*}}, k, \varepsilon\right)\right\rangle \\
& \times \frac{e}{M_{B^{*}}^{2}-k^{2}}\left\langle B^{*-}\left(M_{B^{*}}, k, \varepsilon\right)\left|\bar{b} \gamma_{\nu} b\right| B^{-}\left(M_{1}, p\right)\right\rangle .
\end{aligned}
$$

Note that the imaginary addition $-i M_{B^{*}} \Gamma_{B^{*}}$ does not exist in the propagator, as $k^{2}<M_{B^{*}}^{2}$, i.e., the pole of the $B^{*}$ meson is not reached. The contribution from the $\Upsilon(1 S)$ is taken into account effectively when introducing pole parametrization for the form factor $V_{b}\left(q^{2}\right)$. For the variable $k^{2}$ in the kinematically allowed range, the tensor $T_{\nu \mu}^{(b)}(q, k)$ does not have any other poles.

Numerically, the contribution of the process on Fig. 2 to the branching ratio associated with the four-leptonic decay is suppressed comparing to the contribution of the process on Fig. 1 by factor $\left(\Lambda / m_{b}\right)^{2}$, where $m_{b} \sim 5 \mathrm{GeV}$, the mass of $b$ quark, and the parameter $\Lambda \approx 300-500 \mathrm{MeV}$. This follows from the exact equations for the form factors of the rare leptonic radiative decays of $B$ mesons $[15,16]$. Due to the interference between diagrams 1 and 2 near the photonic pole, it is necessary, however, to take into account the contribution of diagram 2 to the full branching ratio.

The bremsstrahlung contribution is described by the diagram in Fig. 3. The bremsstrahlung amplitude has a single pole by $q^{2}$ from the photon propagator. Hence, the tensor $T_{\nu \mu}^{(\text {brem })}(q, k)$ does not have poles by $q^{2}$ and $k^{2}$. It is important to take into account the bremsstrahlung contribution near the pole by $q^{2}$, where the zero-mass approximation may not be fully correct. This contribution should be calculated for nonzero lepton masses.

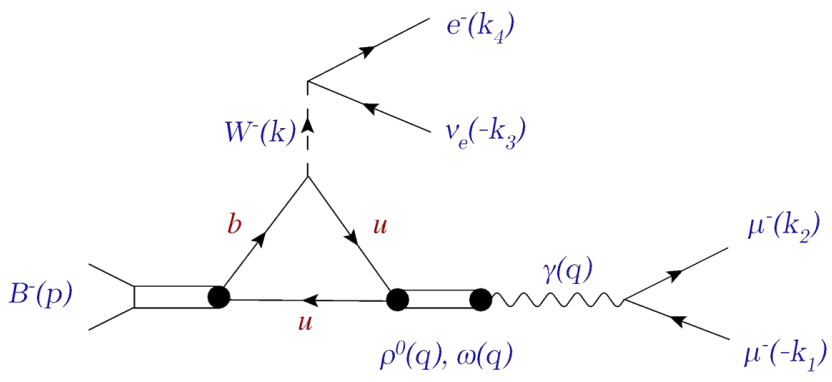

FIG. 4. Diagram for calculation of $\mathcal{M}_{f i}^{(u)}$ [see Eq. (7)] using the decay $B^{-} \rightarrow \mu^{+} \mu^{-} \bar{\nu}_{e} e^{-}$as an example. The emission of the virtual photon by a light quark is described by the vector meson dominance model.

\section{FORMULAS FOR THE DECAY $B^{-} \rightarrow \ell^{+} \ell^{-} \bar{\nu}_{\ell^{\prime}} \ell^{\prime-}$}

Consider the decays $B^{-} \rightarrow \mu^{+} \mu^{-} \bar{\nu}_{e} e^{-}$and $B^{-} \rightarrow$ $e^{+} e^{-} \bar{\nu}_{\mu} \mu^{-}$, for the case when the lepton flavors in the final state are different. Generally, these decays may be written as $B^{-} \rightarrow \ell^{+} \ell^{-} \bar{\nu}_{\ell^{\prime}} \ell^{\prime-}$ for $\ell \neq \ell^{\prime}$.

The contribution to the full decay amplitude $B^{-}(p) \rightarrow$ $\ell^{+}\left(k_{1}\right) \ell^{-}\left(k_{2}\right) \bar{\nu}_{\ell^{\prime}}\left(k_{3}\right) \ell^{\prime-}\left(k_{4}\right)$ from Fig. 1 may be calculated using the vector meson dominance (VMD) model (see Fig. 4). Assuming $m_{\ell}=m_{\ell^{\prime}}=0$ and using the effective Hamiltonian (2), one finds that for VMD the contribution from process (1) is described by diagram 4 , and the corresponding amplitude may be written as

$$
\begin{aligned}
\mathcal{M}_{f i}^{(u)}= & \frac{\mathcal{A}}{q^{2}}\left[\sum_{i=\rho^{0}, \omega} \frac{I_{i} M_{2 i} f_{V_{i}}}{q^{2}-M_{2 i}^{2}+i \Gamma_{2 i} M_{2 i}} \mathcal{F}_{\mu \nu}^{(i)}\left(k^{2}\right)\right] \\
& \times j^{\nu}\left(k_{2}, k_{1}\right) J^{\mu}\left(k_{4}, k_{3}\right),
\end{aligned}
$$

where, using the motion equations,

$$
\begin{aligned}
\mathcal{F}_{\mu \nu}^{(i)}\left(k^{2}\right)= & \frac{2 V^{(i)}\left(k^{2}\right)}{M_{1}+M_{2 i}} \varepsilon_{\mu \nu k q}-i\left(M_{1}+M_{2 i}\right) A_{1}^{(i)}\left(k^{2}\right) g_{\mu \nu} \\
& +2 i \frac{A_{2}^{(i)}\left(k^{2}\right)}{M_{1}+M_{2 i}} q_{\mu} k_{\nu} .
\end{aligned}
$$

For the calculation of the resonances sum in the (7), only the contributions from the lightest $\rho^{0}$ and $\omega$ mesons, containing $u \bar{u}$ pairs, are taken into account. Because the $\rho^{0}$ and $\omega$ mesons are linear combinations of $u \bar{u}$ and $d \bar{d}$ pairs, in order to extract the contributions only $u \bar{u}$ pair alone, an isotopic coefficient $I_{i}$ is introduced. By definition,

$$
I_{\rho^{0}}=\left\langle\rho^{0} \mid \bar{u} u\right\rangle=1 / \sqrt{2}
$$

and 


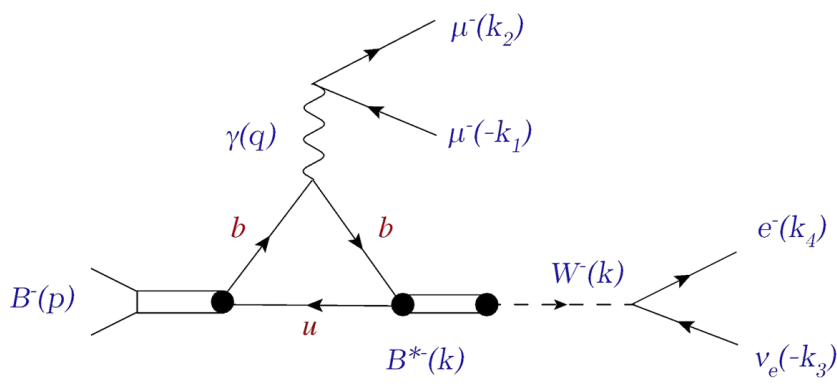

FIG. 5. Diagram for the calculation of $\mathcal{M}_{f i}^{(b)}$ [see Eq. (8)] using the decay $B^{-} \rightarrow \mu^{+} \mu^{-} \bar{\nu}_{e} e^{-}$as an example.

$$
I_{\omega}=\langle\omega \mid \bar{u} u\rangle=1 / \sqrt{2} .
$$

The contribution from process (2) is given by the diagram in Fig. 5, which is the cross-channel of the decay $B^{*} \rightarrow B \gamma^{*}$ of a heavy vector meson into a heavy pseudoscalar meson and a virtual photon, and is represented by

$\mathcal{M}_{f i}^{(b)}=\frac{2}{3} \frac{\mathcal{A}}{q^{2}} \frac{M_{B^{*}} f_{B^{*}}}{k^{2}-M_{B^{*}}^{2}} \frac{V_{b}\left(q^{2}\right)}{M_{1}+M_{B^{*}}} \varepsilon_{\mu \nu k q} j^{\nu}\left(k_{2}, k_{1}\right) J^{\mu}\left(k_{4}, k_{3}\right)$.

There is no imaginary correction in the propagator, as $k^{2}<M_{B^{*}}^{2}$.

Finally, the contribution of the bremsstrahlung process (3) of the virtual photon is described by the diagram in Fig. 6. In the case when $m_{\ell} \neq 0$ and $m_{\ell^{\prime}} \neq 0$, for the amplitude of the bremsstrahlung is

$$
\mathcal{M}_{f i}^{(\text {brem })}=\frac{\mathcal{A}}{q^{2}} i f_{B_{u}} g_{\mu \nu} j^{\nu}\left(k_{2}, k_{1}\right) \tilde{J}^{\mu}\left(k_{4}, k_{3}\right),
$$

where

$$
\begin{aligned}
\tilde{J}^{\mu}\left(k_{4}, k_{3}\right)= & J^{\mu}\left(k_{4}, k_{3}\right)+\frac{m_{\ell^{\prime}}}{\left(p-k_{3}\right)^{2}-m_{\ell^{\prime}}^{2}} \\
& \times\left(\bar{\ell}^{\prime}\left(k_{4}\right) \gamma^{\mu}\left(\hat{p}+m_{\ell^{\prime}}\right)\left(1-\gamma^{5}\right) \nu_{\ell^{\prime}}\left(-k_{3}\right)\right) .
\end{aligned}
$$

As $\left(2 m_{\ell}+m_{\ell^{\prime}}\right)^{2} \leq\left(p-k_{3}\right)^{2} \leq M_{1}^{2}$, the second summand does not contain any poles in the whole kinematically allowed range. The second summand may be compatible with the first one only in the range where $\left(p-k_{3}\right)^{2} \sim$ $\left(2 m_{\ell}+m_{\ell^{\prime}}\right)^{2}$. But this range is suppressed by the phase

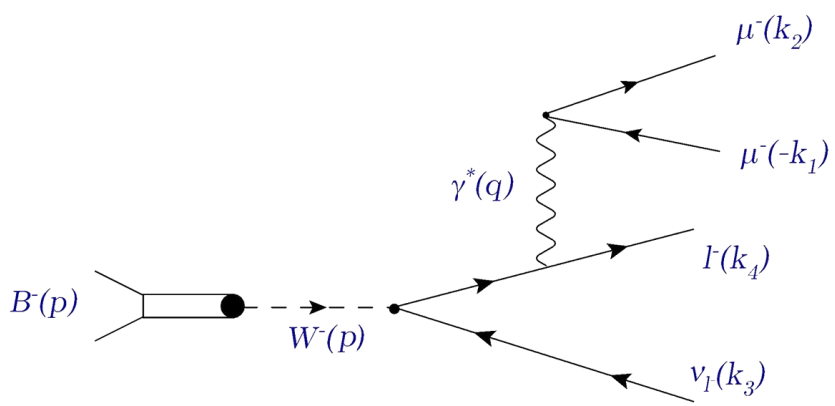

FIG. 6. Diagram for the calculation of the amplitude of the bremsstrahlung $\mathcal{M}_{f i}^{(\mathrm{brem})}$ [see Eq. (9)] for the decay $B^{-} \rightarrow \mu^{+} \mu^{-} \bar{\nu}_{e} e^{-}$.

space (B4) integration. For this the reason, we assume that the bremsstrahlung amplitude may be written as

$$
\mathcal{M}_{f i}^{(\text {brem })}=\frac{\mathcal{A}}{q^{2}} i f_{B_{u}} g_{\mu \nu} j^{\nu}\left(k_{2}, k_{1}\right) J^{\mu}\left(k_{4}, k_{3}\right) .
$$

In formulas (7)-(9), we denote $\mathcal{A}=\frac{G_{F}}{\sqrt{2}} 4 \pi \alpha_{e m} V_{u b}$.

The full decay amplitude $B^{-}(p) \rightarrow \ell^{+}\left(k_{1}\right) \ell^{-}\left(k_{2}\right) \times$ $\bar{\nu}_{\ell^{\prime}}\left(k_{3}\right) \ell^{\prime-}\left(k_{4}\right)$ may be written as

$$
\mathcal{M}_{f i}^{(1234)}=\mathcal{M}_{f i}^{(u)}+\mathcal{M}_{f i}^{(b)}+\mathcal{M}_{f i}^{(\mathrm{brem})} .
$$

The differential branching ratio of the decay $B^{-} \rightarrow$ $\ell^{+} \ell^{-} \bar{\nu}_{\ell^{\prime}} \ell^{\prime-}$ is calculated as

$d \operatorname{Br}\left(B^{-} \rightarrow \ell^{+} \ell^{-} \bar{\nu}_{\ell^{\prime}} \ell^{\prime-}\right)=\tau_{B^{-}} \frac{\sum_{s_{1}, s_{2}, s_{3}, s_{4}}\left|\mathcal{M}_{f i}^{(1234)}\right|^{2}}{2 M_{1}} d \Phi_{4}^{(1234)}$,

where $\tau_{B^{-}}$is the lifetime of the $B^{-}$meson, four-particle phase space $d \Phi_{4}^{(1234)}$ is defined by Eq. (B4), and the summation is performed over the spins of the final fermions. In formula (11), the integration over the angular variables $y_{12}, y_{34}$, and $\varphi$ may be performed analytically [see [10], Eq. (16)]. More accurate and precise values of functions $a\left(x_{12}, x_{34}\right)$, $b\left(x_{12}, x_{34}\right)$, and $c\left(x_{12}, x_{34}\right)$ for calculation of the distribution (16) from [10] are given in Appendix A.

Because in the decay of the $B^{-}$meson, all the leptons in the final state are different, it makes sense to define two forward-backward leptonic asymmetries $A_{F B}^{\left(B^{-}\right)}\left(x_{12}\right)$ and $A_{F B}^{\left(B^{-}\right)}\left(x_{34}\right)$ as

$$
A_{F B}^{\left(B^{-}\right)}\left(x_{12}\right)=\frac{\int_{0}^{1} d \cos \tilde{\theta}_{12} \frac{d^{2} \Gamma\left(B^{-} \rightarrow \ell^{+} \ell^{-} \bar{\nu}_{\ell^{\prime}} \ell^{\prime-}\right)}{d x_{12} d \cos \tilde{\theta}_{12}}-\int_{-1}^{0} d \cos \tilde{\theta}_{12} \frac{d^{2} \Gamma\left(B^{-} \rightarrow \ell^{+} \ell^{-} \bar{\nu}_{\ell^{\prime}} \ell^{\prime-}\right)}{d x_{12} d \cos \tilde{\theta}_{12}}}{\frac{d \Gamma\left(B^{-} \rightarrow \ell^{+} \ell^{-} \bar{\nu}_{\ell^{\prime}} \ell^{\prime-}\right)}{d x_{12}}}
$$

and 


$$
A_{F B}^{\left(B^{-}\right)}\left(x_{34}\right)=\frac{\int_{0}^{1} d \cos \tilde{\theta}_{34} \frac{d^{2} \Gamma\left(B^{-} \rightarrow \ell^{+} \ell^{-} \bar{\nu}_{\ell^{\prime}} \ell^{--}\right)}{d x_{34} d \cos \tilde{\theta}_{34}}-\int_{-1}^{0} d \cos \tilde{\theta}_{34} \frac{d^{2} \Gamma\left(B^{-} \rightarrow \ell^{+} \ell^{-} \bar{\nu}_{\ell^{\prime}} \ell^{\prime-}\right)}{d x_{34} d \cos \tilde{\theta}_{34}}}{\frac{d \Gamma\left(B^{-} \rightarrow \ell^{+} \ell^{-} \bar{\nu}_{\ell^{\prime}} \ell^{\prime-}\right)}{d x_{34}}},
$$

where $\tilde{\theta}_{12}$ is the angle between the propagation directions of the $\ell^{-}$and $B^{-}$in the rest frame of the $\ell^{+} \ell^{-}$pair, and $\tilde{\theta}_{34}$ is the angle between the propagation directions of $\ell^{\prime-}$ and $B^{-}$in the rest frame of the $\ell^{\prime-} \bar{\nu}_{\ell^{\prime}}$ pair. It is obvious that $\tilde{\theta}_{12}=\pi-\theta_{12}$ and $\tilde{\theta}_{34}=\pi-\theta_{34}$. Equations (12) and (13) are chosen such that they correspond to the notions of Ref. [12].

\section{BRANCHING RATIO FORMULAS FOR THE DECAY $B^{-} \rightarrow \ell^{+} \bar{\nu}_{\ell} \ell^{-} \ell^{-}$}

In practice, the muonic tracks are registered with a much higher efficiency at almost all contemporary experiments. That is why from the experimental point of view the decay $B^{-} \rightarrow \mu^{+} \bar{\nu}_{\mu} \mu^{-} \mu^{-}$is of the most interest. In this decay, the final state contains two identical muons of negative charge. Hence, the Fermi antisymmetry should be taken into account.
Consider the full amplitude of the decay $B^{-}(p) \rightarrow$ $\ell^{+}\left(k_{1}\right) \bar{\nu}_{\ell}\left(k_{3}\right) \ell^{-}\left(k_{2}\right) \ell^{-}\left(k_{4}\right)$. In the approximation of zero leptonic masses, the calculation below is applicable to the decay $B^{-} \rightarrow \mu^{+} \bar{\nu}_{\mu} \mu^{-} \mu^{-}$as well as to the decay $B^{-} \rightarrow$ $e^{+} \bar{\nu}_{e} e^{-} e^{-}$. The full amplitude of the decay may be written as

$$
\mathcal{M}_{f i}^{(\mathrm{tot})}=\mathcal{M}_{f i}^{(1234)}-\mathcal{M}_{f i}^{(1432)},
$$

where the amplitude $\mathcal{M}_{f i}^{(1234)}$ is set by Eq. (10), and the amplitude $\mathcal{M}_{f i}^{(1432)}$ can be obtained from $\mathcal{M}_{f i}^{(1234)}$ by exchanging $k_{2} \leftrightarrow k_{4}$. This leads to the necessity of replacing $q_{\mu} \rightarrow \tilde{q}_{\mu}, k_{\mu} \rightarrow \tilde{k}_{\mu}, x_{12} \rightarrow x_{14}$, and $x_{34} \rightarrow x_{23}$ (see Appendix B) in the calculation of $\mathcal{M}_{f i}^{(1432)}$.

The differential branching ratio of the decay is given by

$$
\begin{aligned}
d \operatorname{Br}\left(B^{-} \rightarrow \ell^{+} \bar{\nu}_{\ell} \ell^{-} \ell^{-}\right)= & \frac{1}{2}\left[\tau_{B^{-}} \frac{\sum_{s_{1}, s_{2}, s_{3}, s_{4}}\left|\mathcal{M}_{f i}^{(1234)}\right|^{2}}{2 M_{1}} d \Phi_{4}^{(1234)}+\tau_{B^{-}} \frac{\sum_{s_{1}, s_{2}, s_{3}, s_{4}}\left|\mathcal{M}_{f i}^{(1432)}\right|^{2}}{2 M_{1}} d \Phi_{4}^{(1432)}\right. \\
& \left.-\tau_{B^{-}} \frac{\sum_{s_{1}, s_{2}, s_{3}, s_{4}}\left(\mathcal{M}_{f i}^{(1234) \dagger} \mathcal{M}_{f i}^{(1432)}+\mathcal{M}_{f i}^{(1432) \dagger} \mathcal{M}_{f i}^{(1234)}\right)}{2 M_{1}} d \Phi_{4}^{(1234)}\right],
\end{aligned}
$$

where $d \Phi_{4}^{(1234)}$ and $d \Phi_{4}^{(1432)}$ are set by Eqs. (B4) and (B5). The common factor of $1 / 2$ is due to by Fermi antisymmetry.

The first and the second summands in (15) are equal. Hence, for the branching ratio, it is possible to write

$$
\operatorname{Br}\left(B^{-} \rightarrow \ell^{+} \bar{\nu}_{\ell} \ell^{-} \ell^{-}\right)=\operatorname{Br}\left(B^{-} \rightarrow \ell^{+} \ell^{-} \bar{\nu}_{\ell^{\prime}} \ell^{\prime-}\right)-\operatorname{Br}_{\text {interf }}\left(B^{-} \rightarrow \ell^{+} \bar{\nu}_{\ell} \ell^{-} \ell^{-}\right),
$$

where

$$
\mathrm{Br}_{\text {interf }}\left(B^{-} \rightarrow \ell^{+} \bar{\nu}_{\ell} \ell^{-} \ell^{-}\right)=\frac{\tau_{B^{-}}}{4 M_{1}} \int \sum_{s_{1}, s_{2}, s_{3}, s_{4}}\left(\mathcal{M}_{f i}^{(1234) \dagger} \mathcal{M}_{f i}^{(1432)}+\mathcal{M}_{f i}^{(1432) \dagger} \mathcal{M}_{f i}^{(1234)}\right) d \Phi_{4}^{(1234)}
$$

From (17), it follows that in the calculation of the interference contribution it is necessary to perform fivedimension of numerical integration.

In case of two identical leptons of the same sign in the final state, the experimental measurement of forwardbackward leptonic asymmetries becomes problematic. Hence, in the present work, we do not present any predictions for the corresponding symmetries.

\section{NUMERICAL RESULTS}

To calculate the branching ratio, differential distributions, and asymmetries, we use numerical values of the masses, lifetimes, and decay widths of the pseudoscalar and vector mesons, and matrix elements of the CKM matrix from Ref. [14]. The constants $f_{\rho(770)}=154 \mathrm{MeV}$ and $f_{\omega(782)}=46 \mathrm{MeV}$ were calculated in [16].

Parametrizations of the hadronic form factors (3), except the electromagnetic form factor $V_{b}\left(q^{2}\right)$, were obtained in [13] in the framework of the dispersion relation of the constituent quark model. Using the generic formulas from $[17,18]$ in $[10]$, a parametrization for the electromagnetic form factor $V_{b}\left(q^{2}\right)$ was obtained. The same method allows us to obtain the values of the leptonic constants 


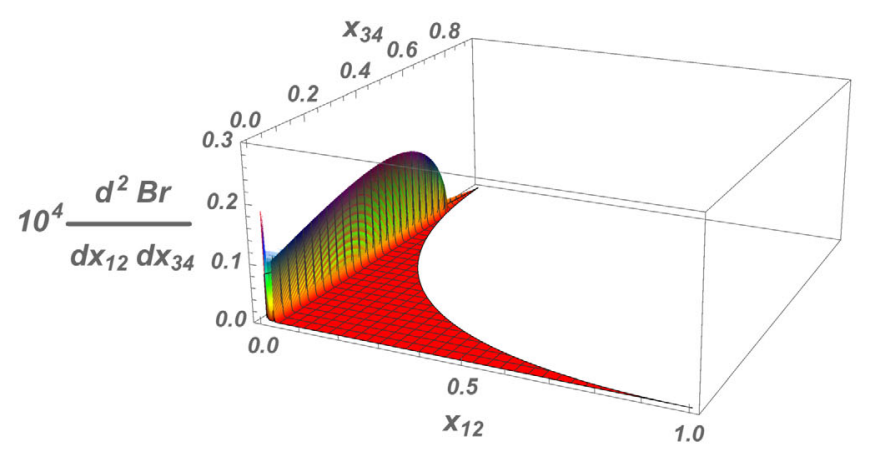

(a)

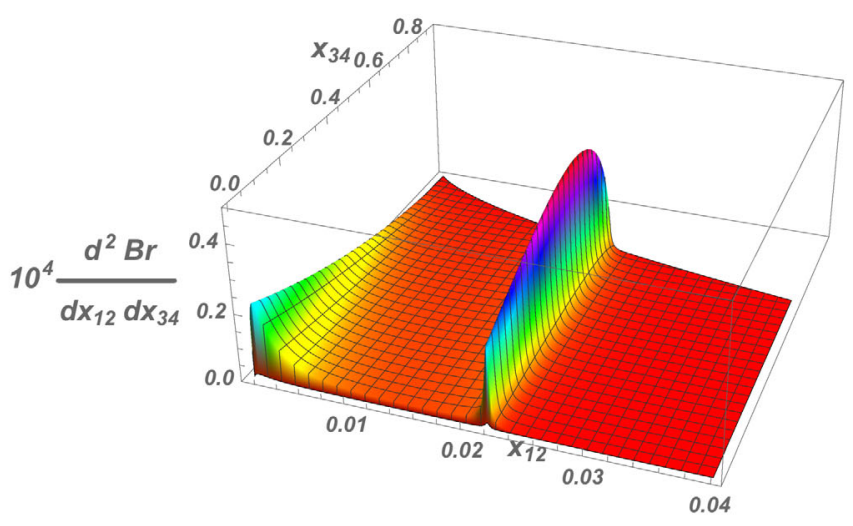

(b)

FIG. 7. Double differential distribution $10^{4} \times \frac{d^{2} \operatorname{Br}\left(B^{-} \rightarrow \mu^{+} \mu^{-} \bar{\nu}_{e} e^{-}\right)}{d x_{12} d x_{34}}$, calculated according to formula (16) from [10] with coefficients that are given in Appendix A. In (b), the range $x_{12} \in[0.00,0.04]$ is highlighted, which corresponds to the area of applicability of the model considered in the present work.

$f_{B_{u}}=191 \mathrm{MeV}$ and $f_{B^{*}}=183 \mathrm{MeV}$. These values of the decay constants and form factors will be used for the calculations below.

We now calculate the branching ratio of the decay $B^{-} \rightarrow \mu^{+} \mu^{-} \bar{\nu}_{e} e^{-}$. The natural kinematical cut of the pole by $x_{12}$ is $x_{12} \min =\left(2 m_{\mu} / M_{1}\right)^{2} \approx 0.0016$. In this case, the numerical integration of Eq. (16) from [10] with more precise coefficients from Appendix A of the present work by $x_{12}$ and $x_{34}$ gives

$$
\begin{aligned}
& \operatorname{Br}\left(B^{-} \rightarrow \mu^{+} \mu^{-} \bar{\nu}_{e} e^{-}\right) \\
& \quad \approx 0.6 \frac{\tau_{B^{-}}}{1.638 \times 10^{-12} \mathrm{~s}} \frac{\left|V_{u b}\right|^{2}}{1.55 \times 10^{-5}} \times 10^{-7} .
\end{aligned}
$$

The value of the branching of the $B^{-} \rightarrow \mu^{+} \mu^{-} \bar{\nu}_{e} e^{-}$decay given in (18) is approximately two times less than the corresponding value of $1.3 \times 10^{-7}$ from Refs. [10,11]. This difference is mostly due to the isotopic coefficients $I_{\rho^{0}}$ and $I_{\omega}$ in (7), while decreases the contribution from the intermediate vector $\rho^{0}(770)$ and $\omega(782)$ resonances to the total branching ratio by a factor 2 . This contribution is dominant, so $\operatorname{Br}\left(B^{-} \rightarrow \mu^{+} \mu^{-} \bar{\nu}_{e} e^{-}\right)$increases by almost the same factor. Also, the mean value of $V_{u b}$ is changed from $4.09 \times 10^{-3}$ [19] to $3.94 \times 10^{-3}$ [14]. A decrease of the branching by $10 \%$ is due to the use of the exact formula (B4) for the phase space.

The result in Eq. (18) is compatible with the naive estimate of (6) up to an expected factor of 2 . The difference between the estimate of (6) and the exact calculation (18) is mostly due to the fact that the estimate does not take into account the pole contribution when $x_{12} \rightarrow x_{12 \mathrm{~min}}$. The importance of the pole contribution becomes obvious when analyzing the double differential distribution $d^{2} \operatorname{Br}\left(B^{-} \rightarrow\right.$ $\left.\mu^{+} \mu^{-} \bar{\nu}_{e} e^{-}\right) / d x_{12} d x_{34}$, which is presented in Fig. 7. The figure features the pole when $x_{12} \rightarrow x_{12} \min =4 m_{\mu}^{2} / M_{1}^{2}$ and the ridge of the narrow $\omega(782)$ resonance, the contribution of which defines the maximum of the matrix element. The wide $\rho^{0}(770)$ meson also gives a significant contribution to the branching ratio, but in the distribution of Fig. 7 is not as prominent as the narrow $\omega(782)$ resonance.

The uncertainty on the numerical value (18) depends on the uncertainty on the calculation of the hadronic form factors of the transitions $B \rightarrow \rho(770)$ and $B \rightarrow \omega(782)$, but does not exceed $20 \%$ [13].

Note that the numerical value of the amplitude of the decay $B^{-} \rightarrow \mu^{+} \mu^{-} \bar{\nu}_{e} e^{-}$significantly depends on the model that describes the contributions of the $\rho^{0}(770)$ and $\omega(782)$ mesons. This dependence is due to the choice of the relative nonperturbative phase $\Phi$ between the contribution of the $\omega(782)$ and $\rho^{0}(770)$ resonances in the amplitude (7). In the framework of the model of VMD, which is used in the present work, phase $\Phi$ is equal to zero. For the phenomenological description of the models with other values of the phase $\Phi$, let us parametrize the amplitude (7) as follows:

$$
\mathcal{M}_{f i}^{(u)}=e^{i \Phi} \mathcal{M}_{f i}^{(\rho)}+\mathcal{M}_{f i}^{(\omega)} .
$$

As the relative phase $\Phi$ is unknown, let us vary the phase in the interval from 0 to $2 \pi$. The results are presented in Table I. One may observe that for $\Phi=\pi$ the branching of the decay $B^{-} \rightarrow \mu^{+} \mu^{-} \bar{\nu}_{e} e^{-}$may decrease by factor of $3 \mathrm{com}-$ paring to the result of (18), obtained for $\Phi=0$. This dependence points to the importance of a future modelindependent study of nonperturbative and nonfactorized contributions of the strong interaction to the amplitudes of the decays $B^{-} \rightarrow \ell^{+} \ell^{-} \bar{\nu}_{\ell^{\prime}} \ell^{\prime-}$. Similar issue of generation of additional relative phases between the contributions of different charmonia by nonfactorizable gluons was discussed in [20].

Below for the calculations of the differential distributions we will work in the framework of VMD, i.e., will imply that 
TABLE I. Dependence of the branching of the decay $B^{-} \rightarrow \mu^{+} \mu^{-} \bar{\nu}_{e} e^{-}$on the relative nonperturbative phase $\Phi$ in the amplitude (19). The first row of the table corresponds to the result of (18).

\begin{tabular}{ll}
\hline \hline Phase $\Phi$ & Branching ratio $\operatorname{Br}\left(B^{-} \rightarrow \mu^{+} \mu^{-} \bar{\nu}_{e} e^{-}\right)$ \\
\hline 0 & $0.6 \frac{\tau_{B^{-}}}{1.638 \times 10^{-12} \mathrm{~s}} \frac{\left|V_{u b}\right|^{2}}{1.55 \times 10^{-5}} \times 10^{-7}$ \\
$\pi / 4$ & $0.5 \frac{\tau_{B^{-}}}{1.638 \times 10^{-12} \mathrm{~s}} \frac{\left|V_{u b}\right|^{2}}{1.55 \times 10^{-5}} \times 10^{-7}$ \\
$\pi / 2$ & $0.3 \frac{\tau_{B^{-}}}{1.638 \times 10^{-12} \mathrm{~s}} \frac{\left|V_{u b}\right|^{2}}{1.55 \times 10^{-5}} \times 10^{-7}$ \\
$3 \pi / 4$ & $0.2 \frac{\tau_{B^{-}}}{1.638 \times 10^{-12} \mathrm{~s}} \frac{\left|V_{u b}\right|^{2}}{1.55 \times 10^{-5}} \times 10^{-7}$ \\
$\pi$ & $0.2 \frac{\tau_{B^{-}}}{1.638 \times 10^{-12} \mathrm{~s}} \frac{\left|V_{u b}\right|^{2}}{1.55 \times 10^{-5}} \times 10^{-7}$ \\
$5 \pi / 4$ & $0.3 \frac{\tau_{B^{-}}}{1.638 \times 10^{-12} \mathrm{~s}} \frac{\left|V_{u b}\right|^{2}}{1.55 \times 10^{-5}} \times 10^{-7}$ \\
$3 \pi / 2$ & $0.5 \frac{\tau_{B^{-}}}{1.638 \times 10^{-12} \mathrm{~s}} \frac{\left|V_{u b}\right|^{2}}{1.55 \times 10^{-5}} \times 10^{-7}$ \\
$7 \pi / 4$ & $0.6 \frac{\tau_{B^{-}}}{1.638 \times 10^{-12} \mathrm{~s}} \frac{\left|V_{u b}\right|^{2}}{1.55 \times 10^{-5}} \times 10^{-7}$ \\
\hline \hline
\end{tabular}

the relative phase $\Phi=0$. Values of $\Phi \neq 0$ will be used only for calculation of the branching of the decay $B^{-} \rightarrow \mu^{+} \mu^{-} \bar{\nu}_{\mu} \mu^{-}$.

In the model used for the result of (18), the nonresonant contribution, which is not related to the tails from the $\rho^{0}(770)$ and $\omega(782)$ resonances, is not taken into account. This contribution may be estimated by using the results from Ref. [21]; in this work, the branching ratio of the decay $B \rightarrow \gamma \ell \nu$ was predicted, omitting the contributions from $\rho^{0}$ and $\omega$ resonances. An estimation of the nonresonant contribution gives

$$
\begin{aligned}
& \operatorname{Br}\left(B^{-} \rightarrow \mu^{+} \mu^{-} \bar{\nu}_{e} e^{-}\right)_{\mathrm{NRC}} \\
& \quad \sim \alpha_{e m} \times \operatorname{Br}(B \rightarrow \gamma \ell \nu)_{\text {Beneke }} \sim 0.1 \times 10^{-7},
\end{aligned}
$$

which is about $15 \%$ of the value of the branching ratio of (18) and is comparable to the uncertainty of the form factors calculation. Note that numerically the contributions to (18) from the processes in Figs. 5 and 6, which were taken into account, are also comparable to the nonresonant contribution, which was not taken into account. The analogous conclusions on the value of nonresonant contribution may be reached if one uses the numerical values $\operatorname{Br}(B \rightarrow \gamma \ell \nu)$ from [22-25].

It seems that the approximation of using only the contributions from the lightest $\rho(770)$ and $\omega(782)$ resonances, which are used in this work, is not applicable if the branching ratio of the decay $B^{-} \rightarrow \mu^{+} \mu^{-} \bar{\nu}_{e} e^{-}$will be measured in the range of $\sqrt{q^{2}}>1 \mathrm{GeV}$. In this range, it is necessary to take into account the contributions from the $\omega(1420), \rho(1450), \omega(1650)$, and $\rho(1700)$ resonances. These contributions should not affect the branching ratio of the decay $B^{-} \rightarrow \mu^{+} \mu^{-} \bar{\nu}_{e} e^{-}$for $\sqrt{q^{2}} \leq 1 \mathrm{GeV}$ but will define the behavior in the range $\sqrt{q^{2}}>1 \mathrm{GeV}$. However, in the experimental procedure [8], the variable $\sqrt{q^{2}}$ is chosen to be less than $980 \mathrm{MeV}$, in order to remove a potential background from the decay $\phi \rightarrow \ell^{+} \ell^{-}$. So, the experimental data are available only in the range of applicability of the current work. This fact allows as to exclude from consideration resonances heavier than the $\rho^{0}(770)$ and $\omega(782)$.

We calculate the branching ratio of the decay $B^{-} \rightarrow$ $e^{+} e^{-} \bar{\nu}_{\mu} \mu^{-}$for $\Phi=0$. Formal integration in the range around the photon pole by $x_{12}$ leads to the rough dependence of the branching on $x_{12 \mathrm{~min}}$,

$$
\mathrm{Br} \sim \int \frac{d x_{12}}{x_{12}^{2}} \sim \frac{1}{x_{12 \min }} .
$$

If we choose $x_{12 \min }=\left(2 m_{e} / M_{1}\right)^{2}$, then by the order of magnitude

$$
\begin{aligned}
\operatorname{Br}\left(B^{-} \rightarrow e^{+} e^{-} \bar{\nu}_{\mu} \mu^{-}\right) & \sim\left(\frac{m_{\mu}}{m_{e}}\right)^{2} \operatorname{Br}\left(B^{-} \rightarrow \mu^{+} \mu^{-} \bar{\nu}_{e} e^{-}\right) \\
& \sim 10^{4} \operatorname{Br}\left(B^{-} \rightarrow \mu^{+} \mu^{-} \bar{\nu}_{e} e^{-}\right) .
\end{aligned}
$$

Because the efficiency of detection of the muonic pairs for $\sqrt{q^{2}}$ below $80-100 \mathrm{MeV}$ is low, this range is not suitable for experimental observation. On the other hand, if we choose $x_{12 \min }=\left(\Lambda / M_{1}\right)^{2}=0.0002$ for $\Lambda=80 \mathrm{MeV}$, then

$$
\begin{aligned}
& \left.\operatorname{Br}\left(B^{-} \rightarrow e^{+} e^{-} \bar{\nu}_{\mu} \mu^{-}\right)\right|_{x_{12 \min }=0.0002} \\
& \quad \approx 3.0 \frac{\tau_{B^{-}}}{1.638 \times 10^{-12} \mathrm{~s}} \frac{\left|V_{u b}\right|^{2}}{1.55 \times 10^{-5}} \times 10^{-7} .
\end{aligned}
$$

The $\operatorname{Br}\left(B^{-} \rightarrow e^{+} e^{-} \bar{\nu}_{\mu} \mu^{-}\right)$will decrease with increasing $x_{12 \min }$.

The decays $B^{-} \rightarrow \mu^{+} \mu^{-} \bar{\nu}_{e} e^{-}$and $B^{-} \rightarrow e^{+} e^{-} \bar{\nu}_{\mu} \mu^{-}$may be suitable for tests of the hypothesis of leptonic universality, if one measures the branching ratio for the fixed value of $x_{12}>\left(2 m_{\mu} / M_{1}\right)^{2}=0.0016$. If the hints for [26-30] violation of the leptonic universality are true, then the ratio between $\operatorname{Br}\left(B^{-} \rightarrow \mu^{+} \mu^{-} \bar{\nu}_{e} e^{-}\right)$and $\operatorname{Br}\left(B^{-} \rightarrow e^{+} e^{-} \bar{\nu}_{\mu} \mu^{-}\right)$may significantly differ from unity [10].

We consider predictions for the branching ratio of the decay $B^{-} \rightarrow \mu^{+} \bar{\nu}_{\mu} \mu^{-} \mu^{-}$at $\Phi=0$, which is more suitable for experimental observation [8], as the efficiency of muon detection is higher than the efficiency of electron detection. Numerical integration of the interference contribution (17) for $x_{12 \min }=\left(2 m_{\mu} / M_{1}\right)^{2}=0.0016$ gives

$$
\begin{aligned}
& \operatorname{Br}_{\text {interf }}\left(B^{-} \rightarrow \mu^{+} \bar{\nu}_{\mu} \mu^{-} \mu^{-}\right) \\
& \quad \approx-0.09 \frac{\tau_{B^{-}}}{1.638 \times 10^{-12} \mathrm{~s}} \frac{\left|V_{u b}\right|^{2}}{1.55 \times 10^{-5}} \times 10^{-7},
\end{aligned}
$$

which is comparable due to uncertainty of the strong nonperturbative effects, the contributions from Eqs. (5) and (6) and the result with the nonresonant contribution 
TABLE II. Dependence of the interference contribution and of the branching of the decay $B^{-} \rightarrow \mu^{+} \bar{\nu}_{\mu} \mu^{-} \mu^{-}$on the relative nonperturbative phase $\Phi$ in the amplitude (19). The first row of the table corresponds to the results of (21) and (22). The third column of the table is obtained by adding up the results of the second column of the table and the last column of Table I according to (16).

\begin{tabular}{lcc}
\hline \hline Phase $\Phi$ & Interference contribution & $\operatorname{Branching} \operatorname{ratio~} \operatorname{Br}\left(B^{-} \rightarrow \mu^{+} \bar{\nu}_{\mu} \mu^{-} \mu^{-}\right)$ \\
\hline 0 & $-0.09 \frac{\tau_{B^{-}}}{1.638 \times 10^{-12} \mathrm{~s}} \frac{\left|V_{u b}\right|^{2}}{1.55 \times 10^{-5}} \times 10^{-7}$ & $0.7 \frac{\tau_{B^{-}}}{1.638 \times 10^{-12} \mathrm{~s}} \frac{\left|V_{u b}\right|^{2}}{1.55 \times 10^{-5}} \times 10^{-7}$ \\
$\pi / 4$ & $-0.10 \frac{\tau_{B^{-}}}{1.638 \times 10^{-12} \mathrm{~s}} \frac{\left|V_{u b}\right|^{2}}{1.55 \times 10^{-5}} \times 10^{-7}$ & $0.6 \frac{\tau_{B^{-}}}{1.638 \times 10^{-12} \mathrm{~s}} \frac{\left|V_{u b}\right|^{2}}{1.55 \times 10^{-5}} \times 10^{-7}$ \\
$\pi / 2$ & $-0.09 \frac{\tau_{B^{-}}}{1.638 \times 10^{-12} \mathrm{~s}} \frac{\left|V_{u b}\right|^{2}}{1.55 \times 10^{-5}} \times 10^{-7}$ & $0.4 \frac{\tau_{B^{-}}}{1.638 \times 10^{-12} \mathrm{~s}} \frac{\left|V_{u b}\right|^{2}}{1.55 \times 10^{-5}} \times 10^{-7}$ \\
$3 \pi / 4$ & $-0.02 \frac{\tau_{B^{-}}}{1.638 \times 10^{-12} \mathrm{~s}} \frac{\left|V_{u b}\right|^{2}}{1.55 \times 10^{-5}} \times 10^{-7}$ & $0.2 \frac{\tau_{B^{-}}}{1.638 \times 10^{-12} \mathrm{~s}} \frac{\left|V_{u b}\right|^{2}}{1.55 \times 10^{-5}} \times 10^{-7}$ \\
$\pi$ & $-0.01 \frac{\tau_{B^{-}}}{1.638 \times 10^{-12} \mathrm{~s}} \frac{\left|V_{u b}\right|^{2}}{1.55 \times 10^{-5}} \times 10^{-7}$ & $0.2 \frac{\tau_{B^{-}}}{1.638 \times 10^{-12} \mathrm{~s}} \frac{\left|V_{u b}\right|^{2}}{1.55 \times 10^{-5}} \times 10^{-7}$ \\
$5 \pi / 4$ & $-0.08 \frac{\tau_{B^{-}}}{1.638 \times 10^{-12} \mathrm{~s}} \frac{\left|V_{u b}\right|^{2}}{1.55 \times 10^{-5}} \times 10^{-7}$ & $0.4 \frac{\tau_{B^{-}}}{1.638 \times 10^{-12} \mathrm{~s}} \frac{\left|V_{u b}\right|^{2}}{1.55 \times 10^{-5}} \times 10^{-7}$ \\
$3 \pi / 2$ & $-0.12 \frac{\tau_{B^{-}}}{1.638 \times 10^{-12} \mathrm{~s}} \frac{\left|V_{u b}\right|^{2}}{1.55 \times 10^{-5}} \times 10^{-7}$ & $0.6 \frac{\tau_{B^{-}}}{1.638 \times 10^{-12} \mathrm{~s}} \frac{\left|V_{u b}\right|^{2}}{1.55 \times 10^{-5}} \times 10^{-7}$ \\
$7 \pi / 4$ & $-0.13 \frac{\tau_{B^{-}}}{1.638 \times 10^{-12} \mathrm{~s}} \frac{\left|V_{u b}\right|^{2}}{1.55 \times 10^{-5}} \times 10^{-7}$ & $0.7 \frac{\tau_{B^{-}}}{1.638 \times 10^{-12} \mathrm{~s}} \frac{\left|V_{u b}\right|^{2}}{1.55 \times 10^{-5}} \times 10^{-7}$ \\
\hline \hline
\end{tabular}

omitted. So, we may state that in the limit of massless leptons, with a $30 \%$ precision from Eqs. (16) and (18), it follows that

$$
\begin{aligned}
& \operatorname{Br}\left(B^{-} \rightarrow \mu^{+} \bar{\nu}_{\mu} \mu^{-} \mu^{-}\right) \\
& \quad \approx 0.7 \frac{\tau_{B^{-}}}{1.638 \times 10^{-12} \mathrm{~s}} \frac{\left|V_{u b}\right|^{2}}{1.55 \times 10^{-5}} \times 10^{-7} .
\end{aligned}
$$

This is obtained for $x_{12 \min }=\left(2 m_{\mu} / M_{1}\right)^{2}=0.0016$. This prediction is almost four times higher than the experimental upper limit (1), obtained in Ref. [8]. What may explain the discrepancy between the experimental result and the theoretical prediction? First, there is quite high uncertainty of the theoretical prediction (22). Second, the value of $\operatorname{Br}\left(B^{-} \rightarrow \mu^{+} \bar{\nu}_{\mu} \mu^{-} \mu^{-}\right)$depends on the relative phase $\Phi$ between the contributions of the $\omega(782)$ and $\rho^{0}(770)$ resonances. In the framework of VMD, $\Phi=0$. The dependence of the interference contribution and of the branching of the decay $B^{-} \rightarrow \mu^{+} \bar{\nu}_{\mu} \mu^{-} \mu^{-}$on nonperturbative phase $\Phi$ is presented in Table II. One may see that the branching of the decay $B^{-} \rightarrow \mu^{+} \bar{\nu}_{\mu} \mu^{-} \mu^{-}$strongly depends on the value of nonperturbative phase $\Phi$. For $\Phi=\pi$, it is numerically close to the upper experimental limit (1), but still slightly exceeds it. However, there are no solid theoretical arguments to favor the value $\Phi=\pi$ over $\Phi=0$. All the other contributions, which were omitted in the current work, could not significantly influence the numerical result of Eq. (22). It seems unlikely that the discrepancy between the prediction and measured result may be attributed to Beyond the Standard Model physics.

Note that the decays $B^{-} \rightarrow e^{+} \bar{\nu}_{e} e^{-} e^{-}$and $B^{-} \rightarrow$ $\mu^{+} \bar{\nu}_{\mu} \mu^{-} \mu^{-}$allow us to introduce yet another test for lepton universality at $x_{12}>0.0016$ and $x_{34}>0.0016$ [10].

We consider single differential distributions for the decays $B^{-} \rightarrow \mu^{+} \mu^{-} \bar{\nu}_{e} e^{-}$and $B^{-} \rightarrow \mu^{+} \bar{\nu}_{\mu} \mu^{-} \mu^{-}$at $\Phi=0$. One-dimensional projections of the double differential distribution $\frac{d^{2} \mathrm{Br}}{d x_{12} d x_{34}}$ by $x_{12}$ and $x_{34}$ are given in Figs. 8 and 9 , respectively. The distributions by $x_{12}$ are given in the range $[0,0.04]$, which corresponds to the area of applicability of the model. Figure 8 features a photon pole for $x_{12} \rightarrow x_{12 \min }=\left(2 m_{\mu} / M_{1}\right)^{2}=0.0016$ and a peak from the

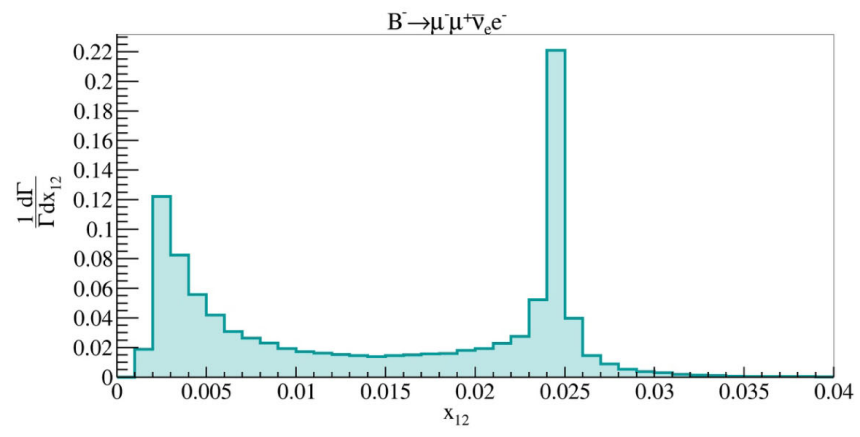

(a)

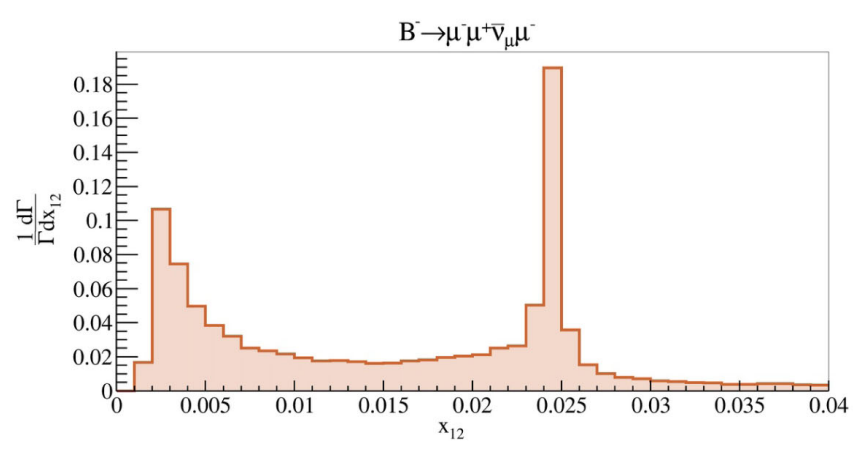

(b)

FIG. 8. Normalized differential distributions $\frac{1}{\Gamma} \frac{d \Gamma}{d x_{12}}$ for the decays (a) $B^{-} \rightarrow \mu^{+} \mu^{-} \bar{\nu}_{e} e^{-}$and (b) $B^{-} \rightarrow \mu^{+} \bar{\nu}_{\mu} \mu^{-} \mu^{-}$, obtained by integration by $d x_{34} d y_{12} d y_{34} d \varphi$ of Eqs. (11) and (15), respectively. 


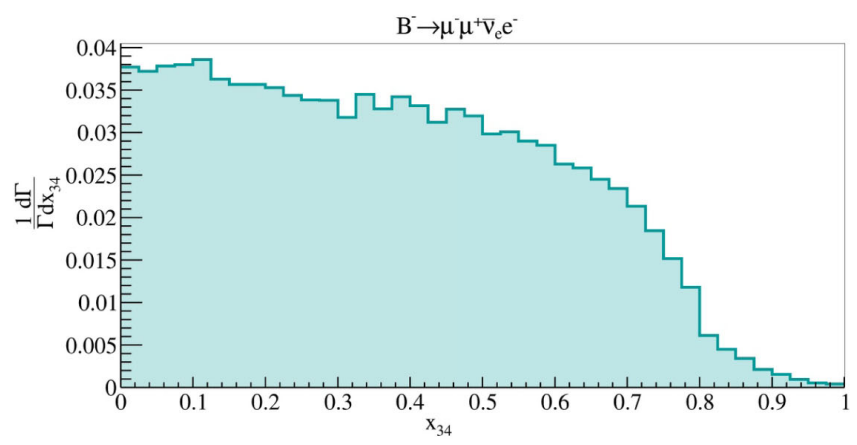

(a)

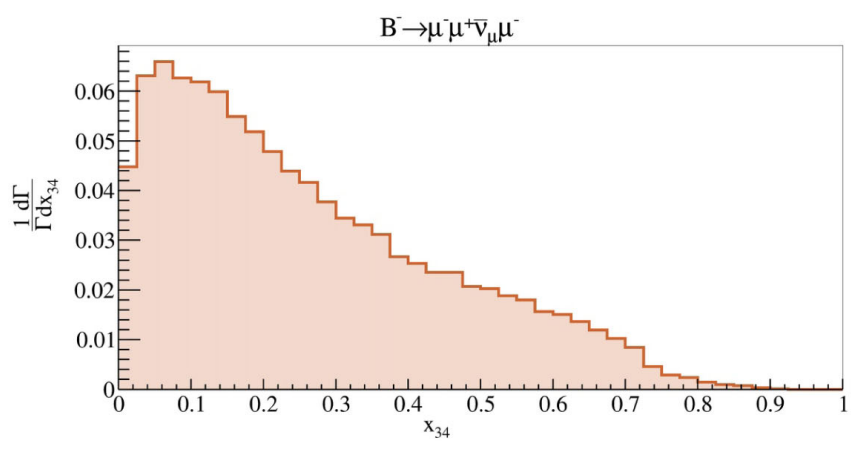

(b)

FIG. 9. Normalized differential distributions $\frac{1}{\Gamma} \frac{d \Gamma}{d x_{34}}$ for the decays (a) $B^{-} \rightarrow \mu^{+} \mu^{-} \bar{\nu}_{e} e^{-}$and (b) $B^{-} \rightarrow \mu^{+} \bar{\nu}_{\mu} \mu^{-} \mu^{-}$, obtained by integration by $d x_{12} d y_{12} d y_{34} d \varphi$ of formulas (11) and (15), respectively.

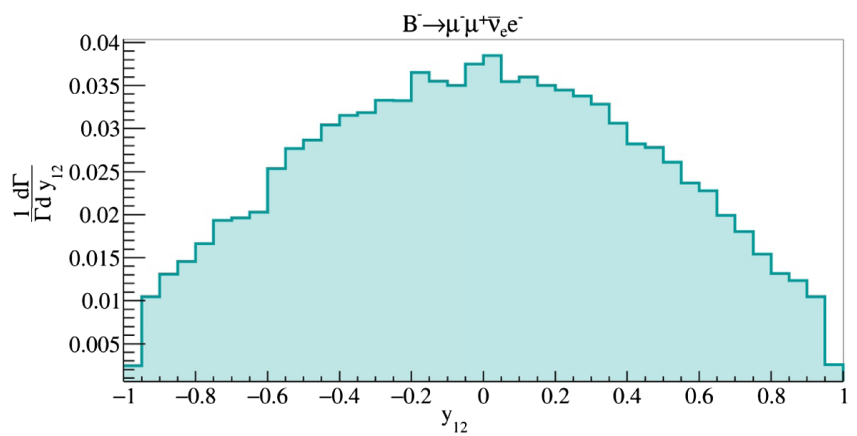

(a)

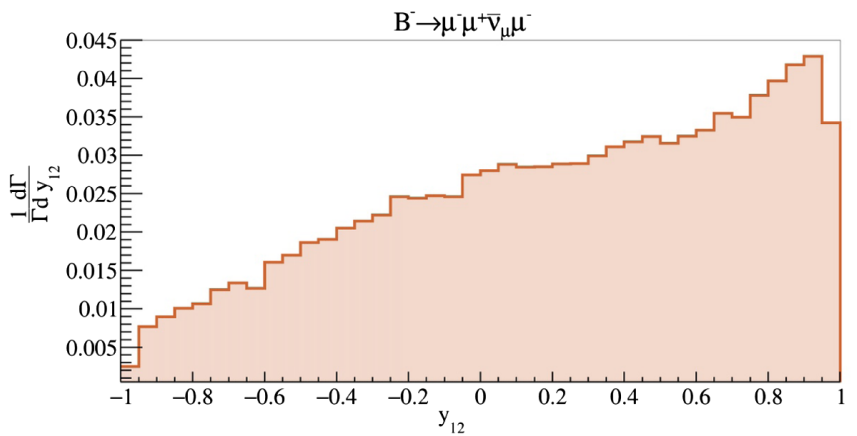

(b)

FIG. 10. Normalized differential distributions $\frac{1}{\Gamma} \frac{d \Gamma}{d y_{12}}$ for the decays (a) $B^{-} \rightarrow \mu^{+} \mu^{-} \bar{\nu}_{e} e^{-}$and (b) $B^{-} \rightarrow \mu^{+} \bar{\nu}_{\mu} \mu^{-} \mu^{-}$, obtained by integrating by $d x_{12} d x_{34} d y_{34} d \varphi$ of formulas (11) and (15) accordingly.

$\omega(782)$ resonance for $x_{12} \rightarrow\left(M_{\omega} / M_{1}\right)^{2} \approx 0.023$. Due to the fact that the $\rho^{0}(770)$ meson has a width of about $150 \mathrm{MeV}$, the contribution from this meson in Fig. 8 appears as a wide background to the narrow peak of the $\omega(782)$ resonance. The distributions by $x_{34}$ in Fig. 9 does not have poles, in agreement with the analysis from Sec. III, and demonstrates the importance of taking into account the Fermi antisymmetry in the decay $B^{-} \rightarrow \mu^{+} \bar{\nu}_{\mu} \mu^{-} \mu^{-}$, because due to the additional contribution from Fermi antisymmetry

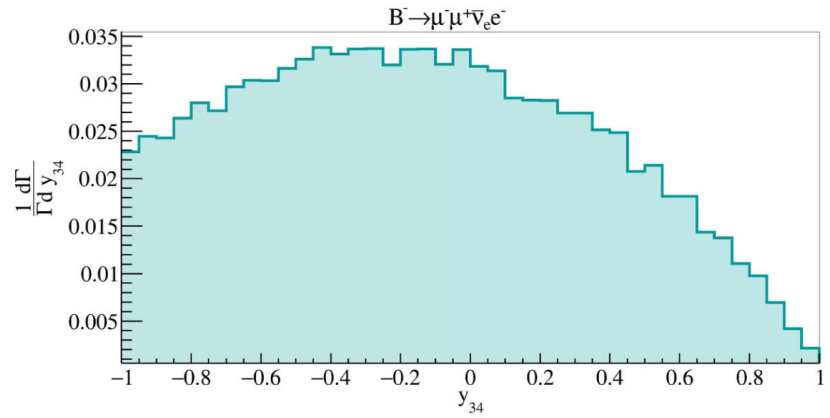

(a) the shapes of the distributions by $x_{34}$ in the decays $B^{-} \rightarrow$ $\mu^{+} \mu^{-} \bar{\nu}_{e} e^{-}$and $B^{-} \rightarrow \mu^{+} \bar{\nu}_{\mu} \mu^{-} \mu^{-}$are significantly different. An analogous difference may be seen in the distributions by $y_{12}=\cos \theta_{12}, y_{34}=\cos \theta_{34}$, and $\varphi$, which are presented in Figs. 10-12, respectively. The definition of angular variables $y_{12}, y_{34}$, and $\cos \varphi$ is given in Appendix B.

Detectability of the multilepton decays of the $B$ mesons with a neutrino in the final state may be linked to the distributions by normalized invariant mass of the

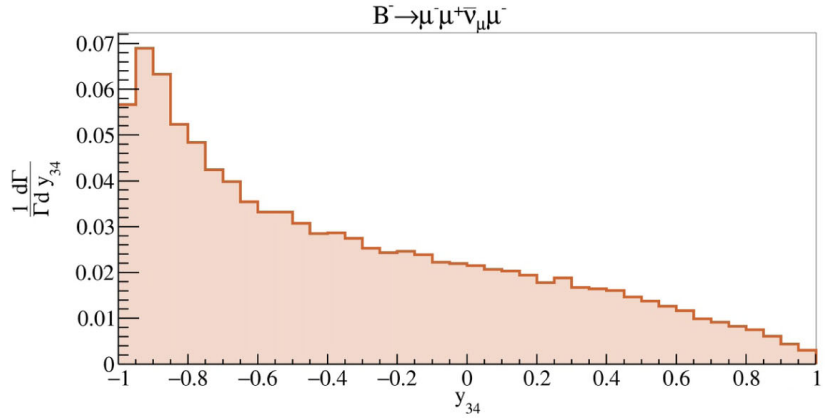

(b)

FIG. 11. Normalized differential distributions $\frac{1}{\Gamma} \frac{d \Gamma}{d y_{34}}$ for the decays (a) $B^{-} \rightarrow \mu^{+} \mu^{-} \bar{\nu}_{e} e^{-}$and (b) $B^{-} \rightarrow \mu^{+} \bar{\nu}_{\mu} \mu^{-} \mu^{-}$, obtained by integration by $d x_{12} d x_{34} d y_{12} d \varphi$ of Eqs. (11) and (15), respectively. 


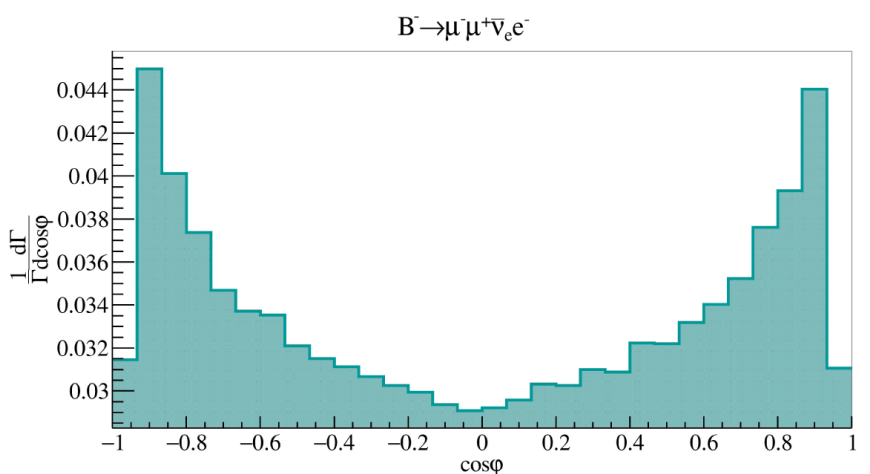

(a)

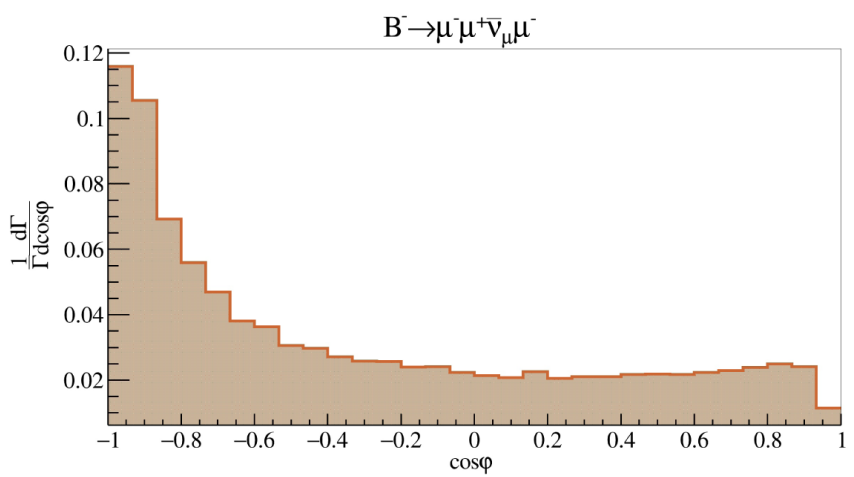

(b)

FIG. 12. Normalized differential distributions $\frac{1}{\Gamma} \frac{d \Gamma}{d \cos \varphi}$ for the decays (a) $B^{-} \rightarrow \mu^{+} \mu^{-} \bar{\nu}_{e} e^{-}$and (b) $B^{-} \rightarrow \mu^{+} \bar{\nu}_{\mu} \mu^{-} \mu^{-}$, obtained by integration by $d x_{12} d x_{34} d y_{12} d y_{34}$ of Eqs. (11) and (15), respectively. Variable $\cos \varphi$ is defined by (B3).

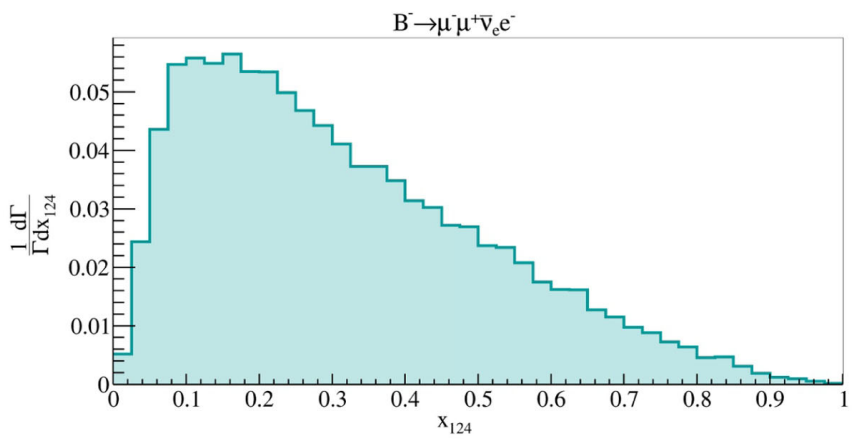

(a)

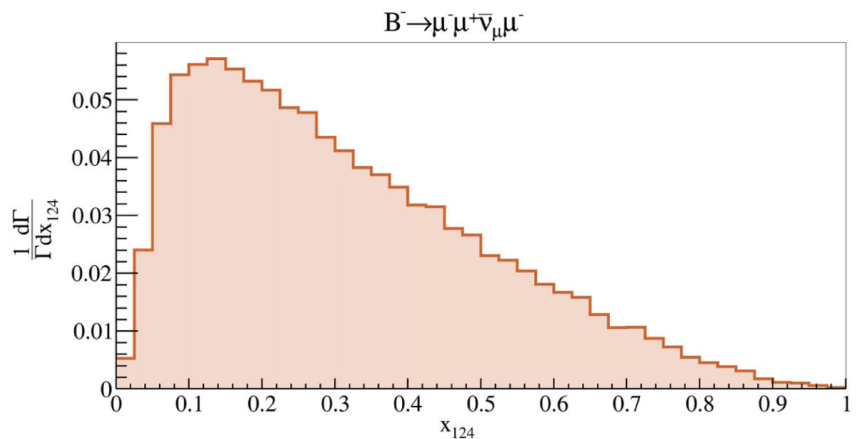

(b)

FIG. 13. Normalized differential distributions $\frac{1}{\Gamma} \frac{d \Gamma}{d x_{124}}$ by invariant mass of all of the charged leptons in the final state for the decays (a) $B^{-} \rightarrow \mu^{+} \mu^{-} \bar{\nu}_{e} e^{-}$and (b) $B^{-} \rightarrow \mu^{+} \bar{\nu}_{\mu} \mu^{-} \mu^{-}$.

charged leptons. The square of the corresponding mass is defined as

$$
x_{124}=\frac{\left(k_{1}+k_{2}+k_{4}\right)^{2}}{M_{1}^{2}},
$$

where $k_{i}$ are four-momenta of charged leptons in the final state. The distributions by $x_{124}$ are presented in Fig. 13. One

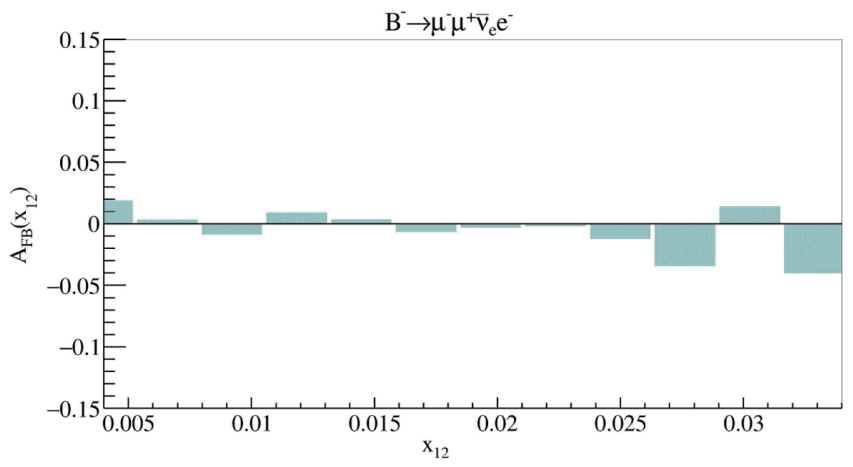

(a) can see from the figure that the shape of the distribution by $x_{12}$ is not very sensitive to the procedure of Fermi antisymmetrization.

It is well known that forward-backward lepton asymmetries are very sensitive to BSM physics. For the decay $B^{-} \rightarrow \mu^{+} \mu^{-} \bar{\nu}_{e} e^{-}$, it is possible to define forward-backward lepton asymmetries $A_{F B}^{\left(B^{-}\right)}\left(x_{12}\right)$ and $A_{F B}^{\left(B^{-}\right)}\left(x_{34}\right)$ according to

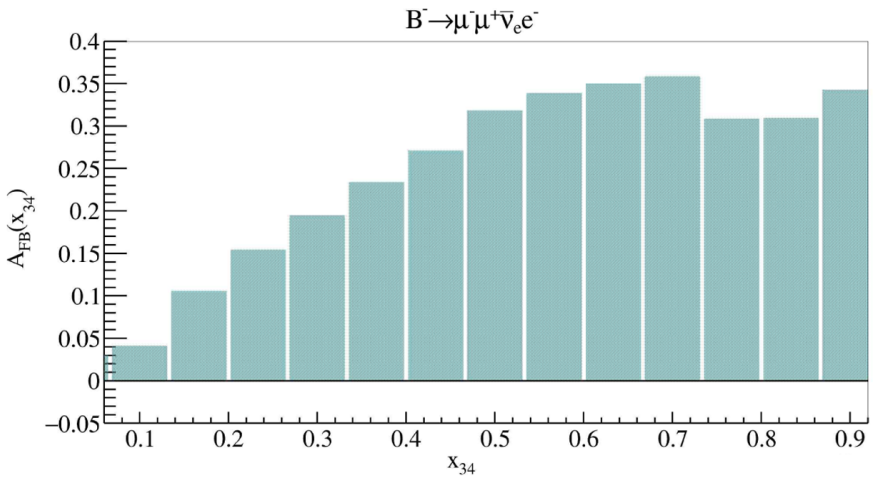

(b)

FIG. 14. Forward-backward lepton asymmetries (a) $A_{F B}^{\left(B^{-}\right)}\left(x_{12}\right)$ and (b) $A_{F B}^{\left(B^{-}\right)}\left(x_{34}\right)$ for the decay $B^{-} \rightarrow \mu^{+} \mu^{-} \bar{\nu}_{e} e^{-}$, calculated using Eqs. (12) and (13), respectively. 
Eqs. (12) and (13). These asymmetries are shown in Fig. 14. The asymmetry $A_{F B}^{\left(B^{-}\right)}\left(x_{12}\right)$ is shown only for the interval $x_{12} \in[0,0.04]$, which corresponds to the area of applicability of the current model. In this interval, excluding the area of the $\omega(782)$ resonance, the contributions to $A_{F B}^{\left(B^{-}\right)}\left(x_{12}\right)$ come from electromagnetic and strong processes; thus, this asymmetry is close to zero in almost all of the considered range. The shape of the asymmetry $A_{F B}^{\left(B^{-}\right)}\left(x_{34}\right)$ is very similar to the shape of the asymmetries in three-body semileptonic decays of $B$ mesons.

One cannot to study forward-backward lepton asymmetries in the decay $B^{-} \rightarrow \mu^{+} \bar{\nu}_{\mu} \mu^{-} \mu^{-}$, as in this case there are two identical negative muons in the final state. Experimentally, it is not possible to distinguish which of the negatively charged muons should be attributed to the $\mu^{+} \mu^{-}$pair and which to the $\bar{\nu}_{\mu} \mu^{-}$pair.

All the above that is related to the differential distributions for the decays $B^{-} \rightarrow \mu^{+} \mu^{-} \bar{\nu}_{e} e^{-}$and $B^{-} \rightarrow \mu^{+} \bar{\nu}_{\mu} \mu^{-} \mu^{-}$ is also related to the differential distributions for the decays $B^{-} \rightarrow e^{+} e^{-} \bar{\nu}_{\mu} \mu^{-}$and $B^{-} \rightarrow e^{+} \bar{\nu}_{e} e^{-} e^{-}$. In this model, the lepton universality holds, so the differential distributions of the two latter decays are not needed.

\section{CONCLUSION}

In the present work,

(i) Theoretical predictions for the branching ratios of the decays $B^{-} \rightarrow \mu^{+} \mu^{-} \bar{\nu}_{e} e^{-}$and $B^{-} \rightarrow \mu^{+} \bar{\nu}_{\mu} \mu^{-} \mu^{-}$for the value of nonperturbative hadronic phase $\Phi=0$ are obtained in the framework of Standard Model,

$$
\operatorname{Br}\left(B^{-} \rightarrow \mu^{+} \mu^{-} \bar{\nu}_{e} e^{-}\right) \approx 0.6 \times 10^{-7}
$$

and

$$
\operatorname{Br}\left(B^{-} \rightarrow \mu^{+} \bar{\nu}_{\mu} \mu^{-} \mu^{-}\right) \approx 0.7 \times 10^{-7},
$$

and uncertainties for every prediction are discussed.

(ii) The difference between the obtained predictions and the predictions from Ref. [10] is discussed, as well as the compatibility with the recent experimental result [8] by the LHCb Collaboration.

(iii) The dependence of branchings of the decays $B^{-} \rightarrow$ $\mu^{+} \mu^{-} \bar{\nu}_{e} e^{-}$and $B^{-} \rightarrow \mu^{+} \bar{\nu}_{\mu} \mu^{-} \mu^{-}$on the nonperturbative phase $\Phi$ is discussed.

(iv) The possibility to test the hypothesis of lepton universality in rare four-leptonic decays of $B$ mesons with three charged leptons in the final state is analyzed.

(v) Double and single differential distributions for the decays $B^{-} \rightarrow \mu^{+} \mu^{-} \bar{\nu}_{e} e^{-}$and $B^{-} \rightarrow \mu^{+} \bar{\nu}_{\mu} \mu^{-} \mu^{-}$are considered, and some recommendations for searches for Beyond the Standard Model physics in these decays are given.

\section{ACKNOWLEDGMENTS}

The authors would like to thank I. M. Belyaev (ITEP), E. E. Boos (SINP MSU), L. V. Dudko (SINP MSU), V. Yu. Yegorychev (ITEP), A. D. Kozachuk (SINP MSU), and D. V. Savrina (ITEP, SINP MSU) for fruitful discussions which improved the current work significantly. The authors would like to especially thank D. I. Melikhov (SINP MSU) for help with calculation of form factor $V_{b}\left(q^{2}\right)$ and numerous fruitful discussions. The authors would like to express their deep gratitude to Professor Sally Seidel (UNM, USA) for help with preparation of the paper. The work was supported by Grant No. 16-12-10280 of the Russian Science Foundation. The authors (A. D. and N.N.) express their gratitude for this support. A. D. is grateful to the "Basis" Foundation for her stipend for Ph.D. students.

\section{APPENDIX A: MORE PRECISE VALUES OF FUNCTIONS $a\left(x_{12}, x_{34}\right), b\left(x_{12}, x_{34}\right)$, AND $c\left(x_{12}, x_{34}\right)$}

More precise dimensionless functions $a\left(x_{12}, x_{34}\right)$, $b\left(x_{12}, x_{34}\right)$, and $c\left(x_{12}, x_{34}\right)$ for calculation of the amplitude (10) of the present work and double differential distribution $d^{2} \operatorname{Br}\left(B^{-} \rightarrow \ell^{+} \ell^{-} \bar{\nu}_{\ell^{\prime}} \ell^{\prime-}\right) / d x_{12} d x_{34}$ from [10] may be written as follows:

$$
\begin{aligned}
a\left(x_{12}, x_{34}\right)= & \frac{1}{3} \frac{\hat{M}_{B^{*}} \hat{f}_{B^{*}}}{x_{34}-\hat{M}_{B^{*}}^{2}} \frac{2 V_{b}\left(M_{1}^{2} x_{12}\right)}{1+\hat{M}_{B^{*}}} \\
& +\sum_{i=\rho^{0}, \omega} \frac{I_{i} \hat{M}_{2 i} \hat{f}_{V_{i}}}{x_{12}-\hat{M}_{2 i}^{2}+i \hat{\Gamma}_{2 i} \hat{M}_{2 i}} \frac{2 V^{(i)}\left(M_{1}^{2} x_{34}\right)}{1+\hat{M}_{2 i}}, \\
b\left(x_{12}, x_{34}\right)= & -\hat{f}_{B_{u}}+\sum_{i=\rho^{0}, \omega} \frac{I_{i} \hat{M}_{2 i} \hat{f}_{V_{i}}}{x_{12}-\hat{M}_{2 i}^{2}+i \hat{\Gamma}_{2 i} \hat{M}_{2 i}}
\end{aligned}
$$

$$
\begin{aligned}
& \times\left(1+\hat{M}_{2 i}\right) A_{1}^{(i)}\left(M_{1}^{2} x_{34}\right), \\
c\left(x_{12}, x_{34}\right)= & \sum_{i=\rho^{0}, \omega} \frac{I_{i} \hat{M}_{2 i} \hat{f}_{V_{i}}}{x_{12}-\hat{M}_{2 i}^{2}+i \hat{\Gamma}_{2 i} \hat{M}_{2 i}} \frac{A_{2}^{(i)}\left(M_{1}^{2} x_{34}\right)}{1+\hat{M}_{2 i}},
\end{aligned}
$$

where the dimensionless variables $x_{12}=q^{2} / M_{1}^{2}$ and $x_{34}=$ $k^{2} / M_{1}^{2}$ are defined in Appendix B, and the dimensionless constants are defined as $\hat{f}_{B_{u}}=f_{B_{u}} / M_{1}, \hat{f}_{B^{*}}=f_{B^{*}} / M_{1}$, $\hat{f}_{V_{i}}=f_{V_{i}} / M_{1}, \hat{M}_{2 i}=M_{2 i} / M_{1}, \hat{M}_{B^{*}}=M_{B^{*}} / M_{1}$, and $\hat{\Gamma}_{2 i}=$ $\Gamma_{2 i} / M_{1}$.

\section{APPENDIX B: KINEMATICS OF FOUR-LEPTON DECAYS}

Denote the four-momenta of the final leptons in fourleptonic decays of $B$ mesons as $k_{i}, i=\{1,2,3,4\}$. Let

$$
\begin{array}{ll}
q=k_{1}+k_{2} ; & k=k_{3}+k_{4} ; \quad \tilde{q}=k_{1}+k_{4} ; \\
\tilde{k}=k_{2}+k_{3} ; & p=k_{1}+k_{2}+k_{3}+k_{4},
\end{array}
$$


where $p$ is the four-momentum of the $B$ meson and $p^{2}=M_{1}^{2}$. For the calculations below, it is suitable to use the dimensionless variables,

$$
x_{12}=\frac{q^{2}}{M_{1}^{2}}, \quad x_{34}=\frac{k^{2}}{M_{1}^{2}}, \quad x_{14}=\frac{\tilde{q}^{2}}{M_{1}^{2}}, \quad x_{23}=\frac{\tilde{k}^{2}}{M_{1}^{2}} .
$$

By common notation, $x_{i j}=\left(k_{i}+k_{j}\right)^{2} / M_{1}^{2}$. Hence, $x_{i j}=x_{j i}$. The leptons may be considered as massless in almost all of the calculations of the present work, i.e., $k_{i}^{2}=0$. However, during the calculation of the bremsstrahlung contribution in the area $q^{2} \sim 4 m_{\ell}^{2}$, where $m_{\ell}$ is the mass of any of the charged leptons of the $\ell^{+} \ell^{-}$pair, it is necessary to take into account the dependence of the bremsstrahlung matrix element and phase space on the value of $m_{\ell}$.

From the conservation law of four-momentum, in the zero-mass limit the variables $x_{i j}$ are linked by

$$
x_{12}+x_{13}+x_{14}+x_{23}+x_{24}+x_{34}=1 .
$$

Let us find the intervals for $x_{i j}$ using the inequality $\left(p_{1} p_{2}\right) \geq \sqrt{p_{1}^{2} p_{2}^{2}}$; then any $x_{i j} \geq 0$. On the other hand, $1 \geq\left(\sqrt{x_{12}}+\sqrt{x_{34}}\right)^{2}$. As $0 \leq x_{34}$, so $x_{12} \leq 1$, and $x_{12} \in[0,1]$. The upper limit of the variable $x_{34}$ depends on the value of $x_{12}: x_{34} \leq\left(1-\sqrt{x_{12}}\right)^{2}$. Thus, for the fixed value of $x_{12}$, the variable $x_{34} \in\left[0,\left(1-\sqrt{x_{12}}\right)^{2}\right]$. For the pair $x_{14}$ and $x_{23}$, the analogous condition holds $x_{14} \in[0,1]$ and for the fixed $x_{14}, x_{23} \in\left[0,\left(1-\sqrt{x_{14}}\right)^{2}\right]$.

Consider the kinematics of the decay $B^{-}(p) \rightarrow$ $\ell^{+}\left(k_{1}\right) \ell^{-}\left(k_{2}\right) \bar{\nu}_{\ell^{\prime}}\left(k_{3}\right) \ell^{\prime-}\left(k_{4}\right)$, when the flavor of negatively charged lepton $\ell^{-}\left(k_{2}\right)$ is different from the flavor of the negatively charged lepton $\ell^{\prime-}\left(k_{4}\right)$. Let the positively charged lepton have the momentum $\mathbf{k}_{1}$, and let the antineutrino have the momentum $\mathbf{k}_{3}$. We define an angle $\theta_{12}$ between the momentum of the positively charged lepton and the direction of the $B$ meson ( $z$ axis) in the rest frame of the $\ell^{+} \ell^{-}$pair, and another angle $\theta_{34}$ between

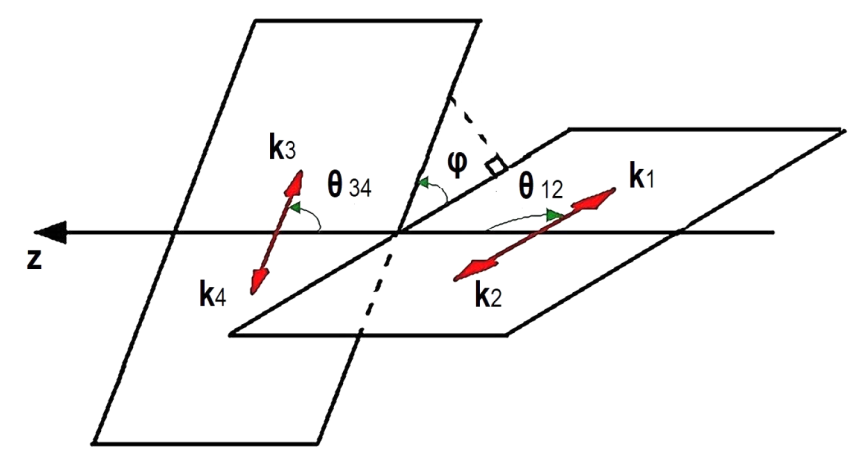

FIG. 15. Kinematics of the decay $B^{-}(p) \rightarrow \ell^{+}\left(k_{1}\right) \times$ $\ell^{-}\left(k_{2}\right) \bar{\nu}_{\ell^{\prime}}\left(k_{3}\right) \ell^{\prime-}\left(k_{4}\right)$. Angle $\theta_{12}$ is defined in the rest frame of $\ell^{+}\left(k_{1}\right) \ell^{-}\left(k_{2}\right)$ pair; angle $\theta_{34}$ is defined in the rest frame of $\bar{\nu}_{\ell^{\prime}}\left(k_{3}\right) \ell^{\prime-}\left(k_{4}\right)$ pair; angle $\varphi$ is defined in the rest frame of $B^{-}$ meson. the direction of the antineutrino and the direction of the $B$ meson ( $z$ axis) in the rest frame of $\ell^{\prime-} \bar{\nu}_{\ell^{\prime}}$ pair, as it is shown in Fig. 15. Then,

$$
\begin{aligned}
& y_{12} \equiv \cos \theta_{12}=\frac{1}{\lambda^{1 / 2}\left(1, x_{12}, x_{34}\right)}\left(x_{23}+x_{24}-x_{13}-x_{14}\right), \\
& y_{34} \equiv \cos \theta_{34}=\frac{1}{\lambda^{1 / 2}\left(1, x_{12}, x_{34}\right)}\left(x_{14}+x_{24}-x_{13}-x_{23}\right),
\end{aligned}
$$

where $\lambda(a, b, c)=a^{2}+b^{2}+c^{2}-2 a b-2 a c-2 b c$, the triangle function. Angles $\theta_{12} \in[0, \pi]$ and $\theta_{34} \in[0, \pi]$. Hence, $y_{12} \in[-1,1]$ and $y_{34} \in[-1,1]$. Angles are measured relative to $z$ axis. Also let us define an angle $\varphi \in$ $[0,2 \pi)$ in the rest frame of the $B$ meson between the planes which are set by the pairs of vectors $\left(\mathbf{k}_{1}, \mathbf{k}_{2}\right)$ and $\left(\mathbf{k}_{3}, \mathbf{k}_{4}\right)$. Introduce a vector $\mathbf{a}_{1}=\mathbf{k}_{1} \times \mathbf{k}_{2}$, perpendicular to the plane $\left(\mathbf{k}_{1}, \mathbf{k}_{2}\right)$, and vector $\mathbf{a}_{3}=\mathbf{k}_{4} \times \mathbf{k}_{3}$, perpendicular to the plane $\left(\mathbf{k}_{3}, \mathbf{k}_{4}\right)$. Then,

$$
\cos \varphi=\frac{\left(\mathbf{a}_{1}, \mathbf{a}_{3}\right)}{\left|\mathbf{a}_{1}\right|\left|\mathbf{a}_{3}\right|} .
$$

Using the technique from Ref. [31], for $\cos \varphi$, we can write

$$
\begin{aligned}
& -2 \sqrt{x_{12} x_{34}\left(1-y_{12}^{2}\right)\left(1-y_{34}^{2}\right)} \cos \varphi+\left(1-x_{12}-x_{34}\right) y_{12} \\
& =x_{13}-x_{14}-x_{23}+x_{24} .
\end{aligned}
$$

Four-particle phase space has the form

$$
d \Phi_{4}^{(1234)}=M_{1}^{4} \frac{d x_{12}}{2 \pi} \frac{d x_{34}}{2 \pi} d \Phi_{2}^{(q k)} d \Phi_{2}^{(12)} d \Phi_{2}^{(34)},
$$

where (assuming nonzero masses for leptons $\ell^{ \pm}$and $\ell^{\prime-}$ ) we can write

$$
\begin{aligned}
d \Phi_{4}^{(1234)}= & \frac{M_{1}^{4}}{2^{14} \pi^{6}} \lambda^{1 / 2}\left(1, x_{12}, x_{34}\right) \sqrt{1-\frac{4 \hat{m}_{\ell}^{2}}{x_{12}}}\left(1-\frac{\hat{m}_{\ell^{\prime}}^{2}}{x_{34}}\right) \\
& \times d x_{12} d x_{34} d y_{12} d y_{34} d \varphi,
\end{aligned}
$$

where $\hat{m}_{\ell}=m_{\ell} / M_{1}$ and $\hat{m}_{\ell^{\prime}}=m_{\ell^{\prime}} / M_{1}$.

In the decay $B^{-}(p) \rightarrow \ell^{+}\left(k_{1}\right) \ell^{-}\left(k_{2}\right) \bar{\nu}_{\ell}\left(k_{3}\right) \ell^{-}\left(k_{4}\right)$, there are two identical leptons $\ell^{-}\left(k_{2}\right)$ and $\ell^{-}\left(k_{4}\right)$ in the final state, so Fermi antisymmetrization of the decay amplitude is necessary by four-momenta $k_{2}$ and $k_{4}$. We will need an additional formula to calculate of the branching ratio in this case for $m_{\ell} \neq 0$,

$$
\begin{aligned}
d \Phi_{4}^{(1432)}= & \frac{M_{1}^{4}}{2^{14} \pi^{6}} \lambda^{1 / 2}\left(1, x_{14}, x_{23}\right) \sqrt{1-\frac{4 \hat{m}_{\ell}^{2}}{x_{12}}}\left(1-\frac{\hat{m}_{\ell}^{2}}{x_{34}}\right) \\
& \times d x_{14} d x_{23} d y_{14} d y_{23} d \tilde{\varphi},
\end{aligned}
$$


where $\tilde{\varphi}$ is the angle of planes $\left(\mathbf{k}_{1}, \mathbf{k}_{4}\right)$ and $\left(\mathbf{k}_{2}, \mathbf{k}_{3}\right)$, measured relative to plane $\left(\mathbf{k}_{1}, \mathbf{k}_{4}\right)$. Equation (B5) may be obtained in a fully analogous way to (B4). The $\cos \tilde{\varphi}$ may be found by exchanging indices in Eq. (B3) as $2 \leftrightarrow 4$. Also, in order to perform numerical integration, it is necessary to have all the definitions of $x_{i j}$ using the set of variables $x_{12}, x_{34}, y_{12}, y_{34}$, and $\varphi$. In the zero leptonic mass limit, the formulas for $x_{13}, x_{14}, x_{23}$, and $x_{24}$ are given in [10]. Finally, note that this paper uses notations almost identical to the notations of Ref. [32], except for in case of the $y_{i j}$, which here have the opposite sign compared to Ref. [32].
[1] V. Khachatryan et al. (CMS and LHCb Collaborations), Observation of the rare $B_{s}^{0} \rightarrow \mu^{+} \mu^{-}$decay from the combined analysis of CMS and LHCb data, Nature (London) 522, 68 (2015).

[2] M. Aaboud et al. (ATLAS Collaboration), Study of the rare decays of $B_{s}^{0}$ and $B^{0}$ into muon pairs from data collected during the LHC Run 1 with the ATLAS detector, Eur. Phys. J. C 76, 513 (2016).

[3] R. Aaij et al. (LHCb Collaboration), Measurement of the $B_{s}^{0} \rightarrow \mu^{+} \mu^{-}$Branching Fraction and Effective Lifetime and Search for $B^{0} \rightarrow \mu^{+} \mu^{-}$Decays, Phys. Rev. Lett. 118, 191801 (2017).

[4] M. Aaboud et al. (ATLAS Collaboration), Study of the rare decays of $B_{s}^{0}$ and $B^{0}$ mesons into muon pairs using data collected during 2015 and 2016 with the ATLAS detector, J. High Energy Phys. 04 (2019) 098.

[5] A. M. Sirunyan et al. (CMS Collaboration), Measurement of properties of $B_{s}^{0} \rightarrow \mu^{+} \mu^{-}$decays and search for $B^{0} \rightarrow \mu^{+} \mu^{-}$ with the CMS experiment, arXiv:1910.12127.

[6] R. Aaij et al. (LHCb Collaboration), Search for Rare $B_{(s)}^{0} \rightarrow$ $\mu^{+} \mu^{-} \mu^{+} \mu^{-}$Decays, Phys. Rev. Lett. 110, 211801 (2013).

[7] R. Aaij et al. (LHCb Collaboration), Search for decays of neutral beauty mesons into four muons, J. High Energy Phys. 03 (2017) 001.

[8] R. Aaij et al. (LHCb Collaboration), Search for the rare decay $B^{+} \rightarrow \mu^{+} \mu^{-} \mu^{+} \nu_{\mu}$, Eur. Phys. J. C 79, 675 (2019).

[9] Y. Dincer and L. M. Sehgal, Electroweak effects in the double Dalitz decay $B_{s} \rightarrow \ell^{+} \ell^{-} \ell^{\prime+} \ell^{\prime-}$, Phys. Lett. B 556, 169 (2003).

[10] A. V. Danilina and N. V. Nikitin, Four-Leptonic decays of charged and neutral $B$ mesons within the Standard Model, Yad. Fiz. 81, 331 (2018) [Phys. At. Nucl. 81, 347 (2018)].

[11] A. Danilina and N. Nikitin, Differential distributions in rare four-leptonic B-decays, EPJ Web Conf. 191, 02011 (2018).

[12] A. Kozachuk, D. Melikhov, and N. Nikitin, Rare FCNC radiative leptonic $B_{s, d} \rightarrow \gamma l^{+} l^{-}$decays in the standard model, Phys. Rev. D 97, 053007 (2018).

[13] D. Melikhov and B. Stech, Weak form-factors for heavy meson decays: An update, Phys. Rev. D 62, 014006 (2000).

[14] M. Tanabashi et al. (Particle Data Group), Review of particle physics, Phys. Rev. D 98, 030001 (2018).

[15] F. Kruger and D. Melikhov, Gauge invariance and formfactors for the decay $B \rightarrow \gamma \ell^{+} \ell^{-}$, Phys. Rev. D 67, 034002 (2003).

[16] D. Melikhov and N. Nikitin, Rare radiative leptonic decays $B_{d, s} \rightarrow \ell^{+} \ell^{-} \gamma$, Phys. Rev. D 70, 114028 (2004).
[17] D. Melikhov, Form-factors of meson decays in the relativistic constituent quark model, Phys. Rev. D 53, 2460 (1996).

[18] D. Melikhov, Heavy quark expansion and universal formfactors in quark model, Phys. Rev. D 56, 7089 (1997).

[19] C. Patrignani (Particle Data Group), Review of particle physics, Chin. Phys. C 40, 100001 (2016).

[20] J. Lyon and R. Zwicky, Resonances gone topsy turvy-the charm of QCD or new physics in $b \rightarrow s \ell^{+} \ell^{-}$? arXiv:1406. 0566.

[21] M. Beneke and J. Rohrwild, B meson distribution amplitude from $B \rightarrow \gamma \ell \nu$, Eur. Phys. J. C 71, 1818 (2011).

[22] G. Eilam, I. E. Halperin, and R. R. Mendel, Radiative decay $B \rightarrow \ell \nu \gamma$ in the light cone QCD approach, Phys. Lett. B 361, 137 (1995).

[23] N. Barik, S. Naimuddin, P. C. Dash, and S. Kar, Radiative leptonic decay: $B^{-} \rightarrow \mu^{-} \bar{\nu}_{\mu} \gamma$ in a relativistic independent quark model, Phys. Rev. D 77, 014038 (2008).

[24] Y. Y. Charng and H. n. Li, B meson wave function from the $B \rightarrow \gamma \ell \nu$, Phys. Rev. D 72, 014003 (2005)

[25] V. M. Braun and A. Khodjamirian, Soft contribution to $B \rightarrow$ $\gamma \ell \nu_{\ell}$ and the $B$-meson distribution amplitude, Phys. Lett. B 718, 1014 (2013)

[26] R. Aaij et al. (LHCb Collaboration), Test of Lepton Universality Using $B^{+} \rightarrow K^{+} \ell^{+} \ell^{-}$Decays, Phys. Rev. Lett. 113, 151601 (2014).

[27] R. Aaij et al. (LHCb Collaboration), Measurement of the Ratio of Branching Fractions $\mathcal{B}\left(\bar{B}^{0} \rightarrow D^{*+} \tau^{-} \bar{\nu}_{\tau}\right) / \mathcal{B}\left(\bar{B}^{0} \rightarrow\right.$ $D^{*+} \mu^{-} \bar{\nu}_{\mu}$ ), Phys. Rev. Lett. 115, 111803 (2015); 115, 159901(E) (2015).

[28] R. Aaij et al. (LHCb Collaboration), Test of lepton universality with $B^{0} \rightarrow K^{* 0} \ell^{+} \ell^{-}$decays, J. High Energy Phys. 08 (2017) 055.

[29] R. Aaij et al. (LHCb Collaboration), Measurement of the Ratio of the $B^{0} \rightarrow D^{*-} \tau^{+} \nu_{\tau}$ and $B^{0} \rightarrow D^{*-} \mu^{+} \nu_{\mu}$ Branching Fractions Using Three-Prong $\tau$-Lepton Decays, Phys. Rev. Lett. 120, 171802 (2018).

[30] R. Aaij et al. (LHCb Collaboration), Search for LeptonUniversality Violation in $B^{+} \rightarrow K^{+} \ell^{+} \ell^{-}$Decays, Phys. Rev. Lett. 122, 191801 (2019).

[31] E. Byckling and K. Kajantie, Particle Kinematics (John Wiley and Sons, London, New York, Sydney, Toronto, 1973).

[32] A. R. Barker, H. Huang, P. A. Toale, and J. Engle, Radiative corrections to double Dalitz decays: Effects on invariant mass distributions and angular correlations, Phys. Rev. D 67, 033008 (2003). 